\title{
STABILITY AND MONOTONICITY OF DIFFERENCE SCHEMES FOR NONLINEAR SCALAR CONSERVATION LAWS AND MULTIDIMENSIONAL QUASI-LINEAR PARABOLIC EQUATIONS
}

\author{
P. MATUS ${ }^{1}$ AND S. LEMESHEVSKY ${ }^{2}$ \\ Dedicated to Prof. P. Vabishchevich on the occasion of his 55th birthday
}

\begin{abstract}
We have proved the difference analogue of a Bihari-type inequality. Using this inequality, we study the stability in $C$ and monotonicity of the difference schemes approximating initial-boundary value problems for nonlinear conservation laws and multi-dimensional parabolic equations. It has been shown that in the nonlinear case the stability and monotonicity are determined not only by the behavior of the approximate solution but also by its difference derivatives appearing in the nonlinear terms of the equation. The stability estimates are obtained without any assumptions about the properties of the solution and nonlinear coefficients of the differential problem. Here we use restrictions only on input data (initial and boundary conditions and the right-hand side).

The sufficient conditions of the shock wave generation is formulated for input data. For the Riemann problem two exact and stable difference schemes are analyzed.
\end{abstract}

2000 Mathematics Subject Classification: 65M06, 65M12, 65Q05.

Keywords: scalar conservation laws, parabolic equations, nonlinear finite-difference schemes, stability, monotonicity, discrete Bihari-type inequality.

\section{Introduction}

Nonlinear hyperbolic conservation laws and parabolic equations play a central role in science and engineering and in the last few years their mathematical theory, as well as their numerical approximation, have made a significant progress $[7,8,12]$. Owing to the nonlinear nature of these equations such physical effects as shock waves, contact discontinuity, boundary layers, and others can appear beyond some finite time interval even when the input data are very smooth functions [12].

We are convinced that the shock wave generation is only determined by the behavior of the input data of the problem $[12,14]$. In this connection, for one- and multidimensional nonlinear scalar conservation laws we study how the properties of initial and boundary

\footnotetext{
${ }^{1}$ Institute of Mathematics, NAS of Belarus, 11 Surganov Str., 220072 Minsk, Belarus, and Department of Mathematics, the John Paul II Catholic University of Lublin, Al. Raclawickie 14, 20-950 Lublin, Poland. E-mail: matus@im.bas-net.by

${ }^{2}$ Institute of Mathematics, NAS of Belarus, 11 Surganov Str., 220072 Minsk, Belarus. E-mail: svl@im.basnet.by
} 
functions and the right-hand side influence the shock wave generation. Estimates of the time of shock wave generation for the one-dimensional case can be found in $[3,10]$.

In investigating difference schemes, the stability of the approximate solution with respect to small perturbations of the input data is one of the main issues. In the nonlinear case, the main distinguishing feature of the stability investigation is the need to study additionally all difference derivatives in the nonlinear terms of the difference equations [16].

The investigation of stability in the nonlinear case is a very difficult problem. In this case, either the a priori estimates are often obtained for linearized problem or certain restrictions on nonlinear coefficients or on the solution are imposed. The $L_{1}$-stability of nonlinear hyperbolic systems has been proven in [4]. In [1,2], the existence, uniqueness and stability of the difference solutions of one-dimensional viscous gas equations have been proved, as well as the error estimates have been derived. All results have been obtained "globally" with respect to the time and without a priori assumptions on the solution of gas motion equations $[1,2] . L^{\infty}$-stability for isentropic gas has been proven in $[13,17]$. In [17], the conditions for the initial and boundary data have been derived. These conditions ensure stability of the difference scheme.

This paper presents the results of investigating the stability of the solutions of difference schemes approximating nonlinear multi-dimensional scalar conservation laws and quasi-linear parabolic equations. The stability estimates have been proved without any assumptions imposed on the solution and nonlinear coefficients of the differential problems. Only restrictions imposed on input data are used. In contrast to the linear problem, the a priori estimates obtained hold, in general, only up to a certain finite instant of time. Of greate importance for these investigations is the discrete Bihari-type inequality [11,23]. A special case of this inequality is the Gronwall inequality used often for investigating the stability and convergence of the difference schemes for time-dependent problems in the linear case. In this paper, we also analyze the stability of the difference schemes approximating the Riemann problem for the Burgers equation.

In investigating the monotonicity, positiveness of the coefficients of the difference scheme or the fulfillment of the maximum principle are often required [28,29]. In the nonlinear case, such requirements should be imposed not on the difference scheme but on the problem for a perturbation $\delta y=\tilde{y}-y$, where $\tilde{y}$ is the solution of the problem with perturbed input data [8]. Consequently, in this case monotone behavior of the difference derivatives of the approximate solution is required $[21,22]$. Note that the monotonicity is necessary for computing problems with highly varying input data [9].

\section{Differential problem and its properties}

In this section, we consider problems for the first-order hyperbolic equations. We analyze how the input data influence the properties of the considered problems solutions.

1.1. The mixed problem for the one-dimensional transport equation. Consider the initial-boundary value problem for the quasi-linear transport equation

$$
\begin{gathered}
\frac{\partial u}{\partial t}+u \frac{\partial u}{\partial x}=f(t, x), \quad 0<x \leqslant l, \quad 0<t \leqslant T, \\
u(t, 0)=\mu(t), \quad 0<t \leqslant T, \quad u(0, x)=u_{0}(x), \quad 0 \leqslant x \leqslant l .
\end{gathered}
$$

Further assume that $u_{0}(x) \geqslant 0$ for all $0<x \leqslant l, u_{0}(0)=\mu(0)>0$ and $\mu(t)>0, f(t, x) \geqslant 0$ 
for all $0<x \leqslant l$ and $0<t \leqslant T$. Under these conditions the solution $u(t, x)$ of problem (1.1), (1.2) is nonnegative $u(t, x) \geqslant 0$ for all $0<x \leqslant l$ and $0 \leqslant t \leqslant T$.

Note that the right-hand side $f(t, x)$ of equation (1.1) in general can change the sign. At the same time, if $f(t, x) \geqslant-M, M>0$, and $u_{0}(x) \geqslant m_{1}>0, \mu(t) \geqslant m_{2}>0$ for all $0<x \leqslant l$ and $0<t \leqslant T\left(M, m_{1}, m_{2}\right.$ are constants), then the solution $u(t, x)$ of problem (1.1), (1.2) is positive only for some interval $\left[0, T^{*}\right]$. In this case, the upper bound of the time interval $T^{*}$ depends on the constants $M, m_{1}, m_{2}$, namely, $T^{*}=\min \left\{m_{1}, m_{2}\right\} / M$. More accurate bounds of $T^{*}$ can be specified by obtaining lower bounds for $u(t, x)$.

To investigate the properties of the solution of the original problem, it is often necessary to estimate its derivative. Differentiation of equation (1.1) with respect to $x$ yields to the Cauchy problem for the Riccati equation with respect to the derivative $v=\partial u / \partial x$

$$
\begin{gathered}
\frac{d v}{d t}=-v^{2}+F(t), \quad F(t)=\frac{\partial f(t, x(t))}{\partial x}, \\
v(0)=v_{0}= \begin{cases}\mu_{1}(t)=\left(f(t, 0)-\mu^{\prime}(t)\right) / \mu(t), & x(t)=0,0<t \leqslant T, \\
u_{0}^{\prime}(x(0)), & 0 \leqslant x(0) \leqslant l, t=0,\end{cases}
\end{gathered}
$$

where $d \cdot / d t$ is the derivative along the direction $d x / d t=u$, i.e., $d u / d t=\partial u / \partial t+u \partial u / \partial x$. Further assume that $u_{0}(x)$ and $\mu_{1}(t)$ satisfy the consistency conditions $u_{0}^{\prime}(0)=\mu_{1}(0)=$ $\left(f(0,0)-\mu^{\prime}(0)\right) / \mu(0)$.

Let us study how the input data (initial and boundary conditions, the right-hand side) influence the generation of a solution discontinuity (shock wave).

Further we use the following norms:

$$
\begin{aligned}
\|v(t)\|_{\bar{C}}= & \max _{0 \leqslant x(t) \leqslant l}\left|\frac{\partial u(t, x(t))}{\partial x}\right|, \quad\|F(t)\|_{C}=\max _{0<x(t) \leqslant l}\left|\frac{\partial f(t, x(t))}{\partial x}\right|, \\
& \left\|v_{0}\right\|_{C_{\gamma}}=\max \left\{\max _{0<t \leqslant T}\left|\mu_{1}(t)\right|, \max _{0 \leqslant x \leqslant l}\left|u_{0}^{\prime}(x)\right|\right\} .
\end{aligned}
$$

1.1.1. The initial conditions influence the shock wave generation. If $\partial f(t, x) / \partial x=0$ and $\mu_{1}(t)=0$ for all $0<x \leqslant l, 0<t \leqslant T$, then the function $v(t)$ is defined as follows:

$$
v(t)=\frac{v_{0}}{1+t v_{0}} .
$$

It is readily seen that if $u_{0}^{\prime}(x) \geqslant 0$ for all $0<x \leqslant l$, then the derivative of the solution of problem (1.1), (1.2) is always bounded, i.e., $\|\partial u(t, x) / \partial x\|_{\bar{C}} \leqslant\left\|u_{0}^{\prime}(x)\right\|_{\bar{C}}$. If the derivative of the initial function $u_{0}(x)$ is negative at least at one point $x^{*}$ then the derivative of the solution of problem (1.1), (1.2) $v=\partial u(t, x) / \partial x$ goes to infinity at the time-point $t^{*}=-1 / u_{0}^{\prime}\left(x^{*}\right)$, i.e., a shock wave has been generated. If the number of such points is more than one, then, denoting by $\mathscr{N}=\left\{x \geqslant 0: u_{0}^{\prime}(x)<0\right\}$ the set of points where the derivative of the initial function is negative, we can determine the moment of shock wave generation as follows:

$$
t^{*}=\frac{1}{\max _{x \in \mathscr{N}}\left|u_{0}^{\prime}(x)\right|}
$$

Thus, if the right-hand side of equation (1.1) is independent of $x$ and the function $\mu$ from the boundary conditions is such that $\mu^{\prime}(t)=f(t, x)$, we get that a shock wave is generated only when $u_{0}^{\prime}(x)$ is negative at least at one point. 
1.1.2. The boundary condition and the right-hand side influence the shock wave generation. Now suppose that the initial condition satisfies $u_{0}^{\prime}(x) \geqslant 0$ for all $x \geqslant 0$. Moreover, assume that $f(t, x)=0$ for all $0<x \leqslant l, 0<t \leqslant T$. Then the derivative $v=\partial u(t, x) / \partial x$ satisfies the following problem:

$$
\frac{d v}{d t}=-v^{2}, \quad v(0)=v_{0}= \begin{cases}-\mu^{\prime}(t) / \mu(t), & x(t)=0,0<t \leqslant T, \\ u_{0}^{\prime}(x(0)), & 0 \leqslant x(0) \leqslant l, t=0 .\end{cases}
$$

In this case, the solution $v(t)$ is also defined by (1.5). Since $u_{0}^{\prime}(x) \geqslant 0$ for all $0 \leqslant x \leqslant l$ it follows that only the boundary condition can generate a shock wave. If $\mu^{\prime}(t) \leqslant 0$ for all $0<t \leqslant T$, then $v_{0} \geqslant 0$ on the boundary $\gamma=\{(t, x): x=0,0<t \leqslant T\} \cup\{(t, x): 0 \leqslant$ $x \leqslant l, t=0\}$, and a shock wave is not generated. But if the function $\mu^{\prime}(t)>0$ at least at one point and $t_{1}=\min _{\mu(t)>0}\{0<t \leqslant T\}$, then $v(t)$ can go to infinity at the time-point:

$$
t_{1}^{*}=\mu\left(t_{1}\right) / \mu^{\prime}\left(t_{1}\right), \quad t_{1}^{*}>t_{1} .
$$

Now let $u_{0}^{\prime}(x) \geqslant 0,0 \leqslant x \leqslant l, \mu^{\prime}(t) \leqslant 0,0<t \leqslant T$, and $\partial f(t, x(t)) / \partial x<0$ at least at one point of the interval $0<x \leqslant l$. In this case, the derivative of the solution of problem (1.1), (1.2) $v=\partial u(t, x) / \partial x$ satisfies the inhomogeneous Riccati equation (1.3) and the initial condition (1.4). Consider a simple example showing how the right-hand side influences the shock wave generation. Let

$$
f(t, x)=-a^{2} x+f_{1}(t), \quad a=\text { const } \neq 0 .
$$

For such a right-hand side, problem (1.3), (1.4) can be solved exactly and the solution is determined by

$$
v(t)=a \operatorname{tg}\left(-a t+\operatorname{arctg} \frac{v_{0}}{a}\right) .
$$

In this case, a shock wave is generated at a time-point defined as follows:

$$
t_{2}^{*}=\frac{\pi}{2|a|}+\frac{1}{|a|} \operatorname{arctg} \frac{\left\|v_{0}\right\|_{C_{\gamma}}}{|a|}
$$

We have considered how the input data influence the shock wave generation.

To obtain an estimate of the derivative in the general case, we can use the following lemma about integral Bihari-type inequalities and its corollary.

Lemma 1.1 [23]. Let $\phi_{i}, i=1,2$ be continuous and nondecreasing functions on $[0, \infty)$ and positive on $(0, \infty)$ such that $\phi=\phi_{2} / \phi_{1}$ is nondecreasing on $(0, \infty)$. Let $u, g_{i}, i=1,2$ be continuous and nonnegative functions on $\left[t_{0}, T\right]$ and $c>0$ a constant. If

$$
u(t) \leqslant c+\int_{t_{0}}^{t} g_{1}(s) \phi_{1}(u(s)) d s+\int_{t_{0}}^{t} g_{2}(s) \phi_{2}(u(s)) d s,
$$

then for $t \in\left[t_{0}, t_{1}\right]$

$$
u(t) \leqslant \Phi_{2}^{-1}\left[\Phi_{2}\left(c_{1}\right)+\int_{t_{0}}^{t} g_{2}(s) d s\right]
$$


where

$$
\Phi_{1}(u)=\int_{c}^{u} \frac{d v}{\phi_{1}(v)}, \quad u>0 ; \quad \Phi_{2}(u)=\int_{u_{0}}^{u} \frac{d v}{\phi_{2}(v)}, \quad u>0, \quad u_{0}>0
$$

and $t_{1}$ is the largest number such that $t_{1} \geqslant t_{0}$ and for $i=1,2$ :

$$
\left\|g_{i}\right\|_{t_{1}}:=\int_{t_{0}}^{t_{1}} g_{i}(s) d s \leqslant \int_{c_{i-1}}^{\infty} \frac{d v}{\phi_{i}(v)}, \quad c_{0}=c, \quad c_{1}=\Phi_{1}^{-1}\left(\left\|g_{1}\right\|_{t_{1}}\right) .
$$

Corollary 1.1. Let $u(t), f(t), g(t)$ be continuous and nonnegative functions on $\left[t_{0}, T\right]$, and $c>0$ a constant. Moreover, suppose that

$$
u(t) \leqslant c+\int_{t_{0}}^{t} f(s) d s+\int_{t_{0}}^{t} g(s) u^{2}(s) d s, \quad t \geqslant t_{0}
$$

and $t_{1}$ is the largest number such that $t_{1} \geqslant t_{0}$ and

$$
\int_{t_{0}}^{t_{1}} g(s) d s \leqslant \frac{1}{c+\int_{t_{0}}^{t_{1}} f(s) d s}
$$

Then for $t \in\left[t_{0}, t_{1}\right]$ the following inequality holds:

$$
u(t) \leqslant \frac{c+\int_{t_{0}}^{t} f(s) d s}{1-\left(c+\int_{t_{0}}^{t} f(s) d s\right) \int_{t_{0}}^{t} g(s) d s} .
$$

Proof. To prove this statement, we put

$$
\phi_{1}(u(s)) \equiv 1, \quad \phi_{2}(u(s))=u^{2}(s), \quad g_{1}(t)=f(t), \quad g_{2}(t)=g(t)
$$

in the conditions of Lemma 1.1 Then

$$
\begin{gathered}
\Phi_{1}(u)=u-c, \quad \Phi_{1}^{-1}(v)=v+c ; \quad \Phi_{2}(u)=\frac{1}{u_{0}}-\frac{1}{u}, \quad \Phi_{2}^{-1}(v)=\frac{u_{0}}{1-u_{0} v}, \quad u_{0}>0 \\
c_{1}=c+\int_{t_{0}}^{t_{1}} f(s) d s .
\end{gathered}
$$

Using these relations and Lemma 1.1, we obtain inequality (1.10).

Remark 1.1. Note that the well-known Gronwall inequality follows from Lemma 1.1 with $g_{1}(t) \equiv 0, \phi_{2}(u) \equiv 1$.

Applying Corollary 1.1 to the solution of problem (1.3), (1.4) we get

$$
\left(\left\|v_{0}\right\|_{C_{\gamma}}+\int_{0}^{t}\|F(\xi)\|_{C} d \xi\right) t=1
$$


For the given $F(t)$ the solution of equation (1.11) gives a time-point $t^{*}$ to which the derivative of the solution of problem (1.1), (1.2) is always bounded, i.e., the estimate

$$
\|v(t)\|_{\bar{C}} \leqslant \frac{\left\|v_{0}\right\|_{C_{\gamma}}+\int_{0}^{t}\|F(\xi)\|_{C} d \xi}{1-t\left(\left\|v_{0}\right\|_{C_{\gamma}}+\int_{0}^{t}\|F(\xi)\|_{C} d \xi\right)}, \quad t-t^{*} \geqslant \delta_{1}>0
$$

holds. Since $\partial u / \partial x \rightarrow \infty$ as $t \rightarrow t^{*}$, consequently, shock wave can be generated. However, in this case, the moment of discontinuity generation cannot be determined exactly . The timepoint $t_{s}$ of shock wave generation belongs to some interval $t_{s} \in\left[t^{*}, t^{*}\right]$. The above approach allows to determine only the lower bound $t^{*}$ of this interval. To set the upper bound of this $\bar{t}^{*}$, we need to obtain the lower bound of the function $v(t)$.

1.2. Multidimensional scalar conservation law. Now let us study how the initial data influence the generation of discontinuity of the solution in multidimensional case. Namely, we study the behavior of the solution gradient depending on the initial data.

Consider the Cauchy problem for the homogeneous quasi-linear transport equation in the space $\mathbb{R}^{d}, d \geqslant 2$.

$$
\begin{aligned}
\frac{\partial u}{\partial t}+\sum_{i=1}^{d} \alpha_{i} u \frac{\partial u}{\partial x_{i}} & =0, \quad \mathbf{x} \in \mathbb{R}^{d}, \quad t \in(0,+\infty), \\
u(0, \mathbf{x}) & =u_{0}(\mathbf{x}), \quad \mathbf{x} \in \mathbb{R}^{d} .
\end{aligned}
$$

Here $0 \leqslant u_{0}(\mathbf{x}) \leqslant U=$ const for all $\mathbf{x} \in \mathbb{R}^{d}$ and $\alpha_{i}(i=1,2, \ldots, d)$ is a positive constant.

Denote by

$$
\frac{d u}{d t}=\frac{\partial u}{\partial t}+\sum_{i=1}^{d} \alpha_{i} u p_{i}
$$

the derivative along the direction given by the system of equations

$$
\frac{d x_{i}}{d t}=\alpha_{i} u(t, \mathbf{x}), \quad i=1,2, \ldots d,
$$

where $p_{i}=\frac{\partial u}{\partial x_{i}}, i=1,2, \ldots, d$. Then we can rewrite equation (1.12) in the following form:

$$
\frac{d u}{d t}=0
$$

Differentiating equation (1.14) with respect to $\left.x_{k}(k=1,2, \ldots, d)\right)$, we get

$$
\frac{d p_{k}}{d t}=-\sum_{i=1}^{d} \alpha_{i} p_{k} p_{i}, \quad k=1,2, \ldots, d .
$$

Multiplying both sides of the last relation by $\alpha_{k}$ and summing the results in $k$ from 1 to $d$, we obtain the Riccati equation with respect to the function $v(t)=\sum_{i=1}^{d} \alpha_{i} p_{i}(t, \mathbf{x}(t))$ :

$$
\frac{d v}{d t}=-v^{2}
$$


From (1.13) we obtain the initial conditions

$$
v(0)=v_{0}=\sum_{i=1}^{d} \alpha_{i} \frac{\partial u_{0}(\mathbf{x})}{\partial x_{i}}=\vec{\alpha} \cdot \nabla u_{0}(\mathbf{x})
$$

where $\vec{\alpha}=\left(\alpha_{1}, \alpha_{2}, \ldots, \alpha_{d}\right)$ is an element of the space $\mathbb{R}^{d}$, and $\cdot$ denotes the inner product in $\mathbb{R}^{d}$.

The solution of problem (1.15), (1.16) is determined by (1.5). Hence, as for the onedimensional case, we conclude that a shock wave is generated if $\vec{\alpha} \cdot \nabla u_{0}(\mathbf{x})<0$ at least at one point of $\mathbb{R}^{d}$. For finding the moment of shock wave generation we have

$$
t^{*}=\frac{1}{\max _{\mathbf{x} \in \mathscr{N}}\left|\vec{\alpha} \cdot \nabla u_{0}(\mathbf{x})\right|},
$$

where $\mathscr{N}=\left\{\mathbf{x} \in \mathbb{R}^{d}: \vec{\alpha} \cdot \nabla u_{0}(\mathbf{x})<0\right\}$.

\section{Preliminary results}

In this section, we discuss some concepts and results necessary for the further investigation of the difference schemes.

2.1. Stability. In general, the stability of a difference scheme means that its solution continuously depends on the input data [25]. In other words, the computational process is called stable if small errors of input data involve small errors of the solution (increase of errors is limited).

Approximation of problems of mathematical physics yields a family of difference schemes, which can be interpreted as an operator equation with operators acting in linear normalized finite-dimensional spaces [25].

For formal description of the difference scheme it is natural to introduce the families of linear spaces $\mathscr{B}_{h}^{(1)}$ and $\mathscr{B}_{h}^{(2)}$, spaces of grid functions given on grid $\bar{\omega}_{h}$ with vector parameter $h$ provided with norm $|h|>0$. Suppose that $\mathscr{B}_{h}^{(1)}, \mathscr{B}_{h}^{(2)}$ are finite-dimensional spaces whose dimension depends on $h$ and can tend to infinity as $|h| \rightarrow 0$. We say that the family of operator equations

$$
\mathcal{A}_{h}\left(y_{h}\right)=\varphi_{h}, \quad y_{h} \in \mathscr{B}_{h}^{(1)}, \quad \varphi_{h} \in \mathscr{B}_{h}^{(2)},
$$

is a difference scheme. Here $\mathcal{A}_{h}\left(y_{h}\right)$ is the nonlinear operator from $\mathscr{D}\left(\mathcal{A}_{h}\right)=\mathscr{B}_{h}^{(1)}$ to $\mathscr{R}\left(\mathcal{A}_{h}\right) \subseteq$ $\mathscr{B}_{h}^{(2)}$. The operator $\mathcal{A}_{h}$ defines the structure of a concrete difference scheme, $y_{h}$ the solution of the difference scheme, and $\varphi_{h}$ the input data of the problem. For example, for the timedependent problems we have

$$
\varphi_{h}= \begin{cases}f_{h} & \text { is the right-hand side at inner grid points } \\ \mu_{h} & \text { is the boundary conditions at boundary grid points } \\ u_{0 h} & \text { is the initial condition at } t=0\end{cases}
$$

Perturbing the input data $\tilde{\varphi}_{h} \in \mathscr{B}^{\left(2_{h}\right)}$ in $(2.1)$ we get the equation with respect to the perturbed solution $\tilde{y}_{h}$ :

$$
\mathcal{A}_{h}\left(\tilde{y}_{h}\right)=\tilde{\varphi}_{h}
$$


The solution $y_{h}$ of the difference scheme (2.1) is called stable if this solution depends on the input data $\varphi_{h}$ continuously and uniformly with respect to $h$. In other words, there exists a positive constant $M$ independent of the choice of $h$ and $\varphi_{h}, \tilde{\varphi}_{h}$ such that for sufficiently small $|h|<h_{0}$ and for any $\varphi_{h}, \tilde{\varphi}_{h} \in \mathscr{B}_{h}^{(2)}$ the following inequality holds:

$$
\left\|\tilde{y}_{h}-y_{h}\right\|_{\left(1_{h}\right)} \leqslant M\left\|\tilde{\varphi}_{h}-\varphi_{h}\right\|_{\left(2_{h}\right)} .
$$

Here $\|\cdot\|_{\left(1_{h}\right)},\|\cdot\|_{\left(2_{h}\right)}$ are the norms of the spaces $\mathscr{B}_{h}^{(1)}$ and $\mathscr{B}_{h}^{(2)}$ respectively.

To derive a stability estimate of the form of (2.3), we need to obtain a problem for the perturbation of the difference solution $\delta y_{h}=\tilde{y}_{h}-y_{h}$. Subtracting equation (2.1) from (2.2), we get

$$
\mathcal{P}_{h}\left(\delta y_{h}, \tilde{y}_{h}, y_{h}\right)=\delta \varphi_{h}
$$

where, in general, the grid operator $\mathcal{P}_{h}$ depends on $\delta y_{h}$, the solutions $\tilde{y}_{h}, y_{h}$, and their difference derivatives [16]. Thus, to investigate problem (2.4), it is necessary to obtain the corresponding a priori estimates for the solutions of problems (2.1) and (2.2) in the strong norms. R. Courant has stressed this property of investigating the solutions of the nonlinear mathematical physics problems in his book [5]. Practically, this means that we have to prove the existence of a solution.

As is well known [25], the concept of stability is a part of the concept of well-posedness of the difference scheme (2.1): the difference scheme (2.1) is well-posed if for a sufficiently small parameter $h$ and for all input data from a certain admissible family there exists a unique solution $y_{h}$ and this solution is stable.

We stress that from estimate (2.3) it follows that the solution is unique. Suppose $\tilde{\varphi}_{h}=$ $\varphi_{h}, y_{h}=y_{1}, \tilde{y}_{h}=y_{2}\left(y_{1}\right.$ and $y_{2}$ are two different solutions for the same input data of problems (2.1) and (2.2)); then from inequality (2.3) we get $\left\|y_{1}-y_{2}\right\|_{\left(1_{h}\right)} \leqslant M\|0\|_{\left(2_{h}\right)}=0$; whence $y_{1}=y_{2}$. So, in the nonlinear case (as well as in the linear case), the requirement of uniqueness of the solution can be omitted in the definition of the well-posedness.

2.2. Difference approximation and convergence. We say that the difference scheme (2.1) approximates the operator equation

$$
\mathcal{A}(u)=f, \quad u \in \mathscr{B}^{(1)}, \quad f \in \mathscr{B}^{(2)}, \quad \mathcal{A}: \mathscr{B}^{(1)} \rightarrow \mathscr{B}^{(2)},
$$

with order $n>0$ if

$\left\|\varphi_{h}-\mathscr{P}_{h}^{(2)}(f)\right\|_{\left(2_{h}\right)}=\left\|\mathcal{A}_{h}\left(u_{h}\right)-\mathscr{P}_{h}^{(2)}(\mathcal{A}(u))\right\|_{\left(2_{h}\right)}=\left\|\mathcal{A}_{h}\left(\mathscr{P}_{h}^{(1)}(u)\right)-\mathscr{P}_{h}^{(2)}(\mathcal{A}(u))\right\|_{\left(2_{h}\right)}=O\left(|h|^{n}\right)$.

Here the projection operators $\mathscr{P}_{h}^{(1)}: \mathscr{B}^{(1)} \rightarrow \mathscr{B}_{h}^{(1)}$ and $\mathscr{P}_{h}^{(2)}: \mathscr{B}^{(2)} \rightarrow \mathscr{B}_{h}^{(2)}$ such that

$$
\begin{array}{ll}
\mathscr{P}_{h}^{(1)}(u)=u_{h} \in \mathscr{B}_{h}^{(1)}, & \text { if } u \in \mathscr{B}^{(1)}, \\
\mathscr{P}_{h}^{(2)}(f)=f_{h} \in \mathscr{B}_{h}^{(2)}, & \text { if } f \in \mathscr{B}^{(2)},
\end{array}
$$

and

$$
\lim _{|h| \rightarrow 0}\left\|\mathscr{P}_{h}^{(1)}(u)\right\|_{\left(1_{h}\right)}=\|u\|_{(1)}, \quad \lim _{|h| \rightarrow 0}\left\|\mathscr{P}_{h}^{(2)}(f)\right\|_{\left(2_{h}\right)}=\|f\|_{(2)},
$$

where $\|\cdot\|_{(k)}$ is the norm in the space $\mathscr{B}^{(k)}, k=1,2$.

To prove the convergence of the solution $y_{h}$ of the difference scheme (2.1) to the solution of the operator equation (2.5), i.e. to obtain the estimate

$$
\left\|y_{h}-u_{h}\right\|_{\left(1_{h}\right)} \leqslant M|h|^{n}, \quad u_{h}=\mathscr{P}_{h}^{(1)}(u),
$$

with a constant $M>0$ independent of $h$, we can use the following 
Theorem 2.1. If scheme (2.1) is stable and approximates problem (2.5), then the solution $y_{h}$ of problem (2.1) converges to the solution $u \in \mathscr{B}^{(1)}$ of equation (2.5) as $|h| \rightarrow 0$. Moreover, the order of convergence of scheme (2.1) is equal to the the order of the difference approximation.

The proof for linear and nonlinear operator $\mathcal{A}_{h}$ is found in [25].

2.3. Monotonicity of the difference schemes. In the theory of computational methods the concept of monotonicity is very important because monotonicity ensures the absence of nonphysical oscillations in numerical computations. Keeping the property of monotonicity of the difference scheme is very important for problems with highly varying input data [9].

In the linear case, the monotonicity of the difference scheme follows from the positiveness of the coefficients of the difference scheme [9] or from the grid maximum principle $[25,27,28]$. The maximum principle is also fulfilled under positive coefficients of difference scheme (2.1). In the nonlinear case, we need a more precise definition [15]. The most correct and natural definition is given in the monograph [8]. For the abstract problem (2.1) this definition is formulated as follows.

Definition 2.1. The difference scheme (2.1) is called monotone if from the condition $\tilde{\varphi}_{h} \geqslant \varphi_{h}\left(\tilde{\varphi}_{h} \leqslant \varphi_{h}\right)$ it follows that $\tilde{y}_{h} \geqslant y_{h}\left(\tilde{y}_{h} \leqslant y_{h}\right)$ at all grid points $\bar{\omega}_{h}$.

In other words, the difference scheme is monotone if from the condition $\delta \varphi \geqslant 0(\delta \varphi \leqslant 0)$ is follows that $\delta y \geqslant 0(\delta y \leqslant 0)$. This implies that in the nonlinear case problem (2.4) for the investigation of stability is the same as for the investigation of monotonicity. Certainly, the conditions of positiveness of the difference operator coefficients or the fulfillment of the grid maximum principle used in the linear case can also be used for the nonlinear problems. But these conditions have to be applied to the nonlinear problem for perturbation (2.4) mismatched with problem (2.1) or (2.2). Note that in this case the requirement of positiveness of the coefficients leads to the requirement of monotone behavior for the difference derivatives $[16,21,22]$.

2.4. Grid maximum principle. Let $\bar{\omega}_{h}$ be a finite set of points (grid) in a certain bounded domain of the Euclidian space of dimension $d$. The grid point $\mathbf{x} \in \bar{\omega}_{h}$ is called the boundary point of the grid $\bar{\omega}_{h}$ if at this grid point the value $y(\mathbf{x})=\mu(\mathbf{x})$ of the function $y(\mathbf{x})$ is given. The set of all boundary grid points is said to be the boundary of the grid and is denoted by $\partial \omega_{h}$. Other grid points of $\bar{\omega}_{h}$ are called interior grid points and the set of interior grid points is denoted by $\omega_{h}$.

On the grid $\bar{\omega}_{h}$ we consider the difference scheme

$$
\alpha(\mathbf{x}) y(\mathbf{x})=\sum_{\mathbf{s} \in \mathcal{M}(\mathbf{x})} \beta(\mathbf{x}, \mathbf{s}) y(\mathbf{s})+\varphi(\mathbf{x}), \quad \mathbf{x} \in \omega_{h},\left.\quad y\right|_{\mathbf{x} \in \partial \omega_{h}}=\mu(\mathbf{x}), \quad \mathbf{x} \in \partial \omega_{h}
$$

Scheme (2.6) is called the canonical form of difference equations [25]. Here $\alpha(\mathbf{x}), \beta(\mathbf{x}, \mathbf{s})$, $\varphi(\mathbf{x})$ are the grid functions defined for all $\mathbf{x}, \mathbf{s} \in \omega_{h}$. Summation is performed over a certain subset $\mathcal{M}(\mathbf{x})$ of the grid $\bar{\omega}_{h}, \mathcal{M}(\mathbf{x})$ does not contain the grid point $\mathbf{x}$, and $\mathcal{T}(\mathbf{x})=\mathcal{M}(\mathbf{x}) \cup\{\mathbf{x}\}$ is the stencil of the difference scheme. The subset $\mathcal{M}(\mathbf{x})$ is called the neighborhood of the grid point $\mathbf{x}$. Suppose that the grid $\bar{\omega}_{h}$ is connected, i.e., for any two grid points $\overline{\mathbf{x}} \in \bar{\omega}_{h}$ and $\overline{\overline{\mathbf{x}}} \in \bar{\omega}_{h}$ there exist grid points $\mathbf{x}_{1}, \mathbf{x}_{2}, \ldots, \mathbf{x}_{m} \in \bar{\omega}_{h}$ such that

$$
\overline{\mathbf{x}} \in \mathcal{M}\left(\mathbf{x}_{1}\right), \quad \mathbf{x}_{1} \in \mathcal{M}\left(\mathbf{x}_{2}\right), \ldots, \mathbf{x}_{m-1} \in \mathcal{M}\left(\mathbf{x}_{m}\right), \quad \mathbf{x}_{m} \in \mathcal{M}(\overline{\overline{\mathbf{x}}})
$$


Let us estimate the solution $y(\mathbf{x})$ of problem $(2.6)$ in the $C\left(\bar{\omega}_{h}\right)$-norm. The following notation is needed for the sequel

$$
\|y\|_{C\left(\bar{\omega}_{h}\right)}=\max _{\mathbf{x} \in \bar{\omega}_{h}}|y(\mathbf{x})|, \quad\|y\|_{C\left(\omega_{h}\right)}=\max _{\mathbf{x} \in \omega_{h}}|y(\mathbf{x})|
$$

Lemma 2.1. Suppose the following conditions hold:

$$
\begin{aligned}
& \alpha(\mathbf{x}) \geqslant 0, \quad \varphi(\mathbf{x}) \geqslant f>0, \quad \text { for all } \mathbf{x} \in \omega_{h}, \\
& \beta(\mathbf{x}, \mathbf{s}) \geqslant 0, \text { for all } \mathbf{x}, \mathbf{s} \in \omega_{h}, \\
& 0<\gamma(\mathbf{x})=\alpha(\mathbf{x})-\sum_{\mathbf{s} \in \mathcal{M}} \beta(\mathbf{x}, \mathbf{s}) \leqslant d_{2}, \quad \text { for all } \mathbf{x} \in \omega_{h}, \\
& \mu(\mathbf{x}) \geqslant m_{1}>0, \text { for all } \mathbf{x}, \in \partial \omega_{h} .
\end{aligned}
$$

Then

$$
y(\mathbf{x}) \geqslant c_{1}>0 \quad \text { for all } \quad \mathbf{x} \in \bar{\omega}_{h} \quad c_{1}=\min \left\{m_{1}, \frac{f}{d_{2}}\right\} .
$$

Proof. If the function $y(\mathbf{x})$ reaches its minimum on the boundary of the grid $\partial \omega_{h}$, then

$$
y(\mathbf{x}) \geqslant m_{1}>0, \quad \mathbf{x} \in \bar{\omega}_{h} .
$$

If the function $y(\mathbf{x})$ reaches its minimum at some interior grid point $\mathbf{x}=\mathbf{x}_{*} \in \omega_{h}$, then from (2.6) we have

$$
\alpha\left(\mathbf{x}_{*}\right) y\left(\mathbf{x}_{*}\right)=\sum_{\mathbf{s} \in \mathcal{M}} \beta\left(\mathbf{x}_{*}, \mathbf{s}\right) y(\mathbf{s})+\varphi\left(\mathbf{x}_{*}\right) \geqslant \sum_{\mathbf{s} \in \mathcal{M}\left(\mathbf{x}_{*}\right)} \beta\left(\mathbf{x}_{*}, \mathbf{s}\right) y\left(\mathbf{x}_{*}\right)+\varphi\left(\mathbf{x}_{*}\right) .
$$

Whence,

$$
\left(\alpha\left(\mathbf{x}_{*}\right)-\sum_{\mathbf{s} \in \mathcal{M}\left(\mathbf{x}_{*}\right)} \beta\left(\mathbf{x}_{*}, \mathbf{s}\right)\right) y\left(\mathbf{x}_{*}\right) \geqslant \varphi\left(\mathbf{x}_{*}\right) .
$$

Taking into account conditions (2.7) from the last relation, we get

$$
y\left(\mathbf{x}_{*}\right) \geqslant f / d_{2}>0
$$

Combining (2.9) and (2.10), we obtain inequality (2.8).

Lemma 2.2. Suppose that for all $\mathbf{x}, \mathbf{s} \in \omega_{h}$ the following conditions are fulfilled:

$$
\alpha(\mathbf{x}) \geqslant 0, \quad \beta(\mathbf{x}, \mathbf{s}) \geqslant 0, \quad \gamma(\mathbf{x})=\alpha(\mathbf{x})-\sum_{\mathbf{s} \in \mathcal{M}(\mathbf{x})} \beta(\mathbf{x}, \mathbf{s})>0 .
$$

Then

$$
\|y(\mathbf{x})\|_{C\left(\bar{\omega}_{h}\right)} \leqslant \max \left\{\|\mu\|_{C\left(\partial \omega_{h}\right)},\left\|\frac{\varphi}{\gamma}\right\|_{C\left(\omega_{h}\right)}\right\} .
$$

Proof. If the grid function $y(\mathbf{x})$ reaches its maximum on the boundary of the grid $\partial \omega_{h}$, then $\|y\|_{C\left(\bar{\omega}_{h}\right)}=\|\mu\|_{C\left(\partial \omega_{h}\right)}$. But if the grid function $y$ reaches its maximum at an interior grid-point $\mathbf{x}^{*} \in \omega_{h}$, then from (2.6) we get

$$
\alpha\left(\mathbf{x}^{*}\right)\|y\|_{C\left(\bar{\omega}_{h}\right)} \leqslant \sum_{\mathbf{s} \in \mathcal{M}\left(\mathbf{x}^{*}\right)} \beta\left(\mathbf{x}^{*}, \mathbf{s}\right)\|y\|_{C\left(\bar{\omega}_{h}\right)}+\left|\varphi\left(\mathbf{x}^{*}\right)\right| .
$$

Now using (2.11), from the last inequality we get the estimate

$$
\|y\|_{\bar{C}} \leqslant\|\varphi / \gamma\|_{C\left(\omega_{h}\right)} .
$$

Thus we obtain estimate (2.12). 
Lemma 2.3. Suppose the following recurrence relation holds:

$$
\left\|y^{n+1}\right\|_{C\left(\bar{\omega}_{h}\right)} \leqslant \max \left\{\mu^{n+1},\left\|y^{n}\right\|_{C\left(\bar{\omega}_{h}\right)}\right\}+\tau\left\|f^{n+1}\right\|_{C\left(\omega_{h}\right)} .
$$

Then

$$
\begin{gathered}
\left\|y^{n+1}\right\|_{C\left(\bar{\omega}_{h}\right)} \leqslant c_{2 n}, \quad n=0,1, \ldots, \\
c_{2 n}=\max \left\{\max _{0 \leqslant k \leqslant n} \mu^{k+1},\left\|y^{0}\right\|_{C\left(\bar{\omega}_{h}\right)}\right\}+\sum_{k=0}^{n} \tau\left\|f^{k+1}\right\|_{C\left(\omega_{h}\right)} .
\end{gathered}
$$

This lemma can be proved by induction (see [20]).

2.5. Discrete Bihari-type inequality. In section 1, in the general case, we use an integral Bihari-type inequality to obtain estimates of the derivative of the solution. Here we prove a discrete Bihari-type inequality.

Lemma 2.4 (discrete analogue of Corollary 1.1). Let $\mathscr{E}_{n}, \mathscr{G}_{n}, g_{n}$ and $f_{n}$ be nonnegative functions defined on the grid $\omega_{\tau}=\left\{t_{n}=\tau n, n=0,1, \ldots\right\}$. Besides, suppose that $\mathscr{G}_{n+1} \geqslant \mathscr{G}_{n}$ and $\mathscr{G}_{n} \geqslant \mathscr{E}_{0}$ for all $n=1,2, \ldots$ Then, if the inequalities

$$
\begin{gathered}
\mathscr{E}_{n+1} \leqslant \mathscr{G}_{n+1}+\sum_{k=0}^{n} \tau g_{k} \mathscr{E}_{k+1} \mathscr{E}_{k}, \\
\sum_{k=0}^{n} \tau g_{k}<\frac{1}{\mathscr{G}_{n+1}}, \quad n=0,1, \ldots,
\end{gathered}
$$

hold, then

$$
\mathscr{E}_{n+1} \leqslant \frac{\mathscr{G}_{n+1}}{1-\mathscr{G}_{n+1} \sum_{k=0}^{n} \tau g_{k}} .
$$

Proof. The proof is by induction on $n$. For $n=0$, taking into account the properties of the grid function $\mathscr{G}_{n}$, from inequalities (2.15) and (2.16) we have

$$
\mathscr{E}_{1} \leqslant \mathscr{G}_{1}+\tau g_{0} \mathscr{E}_{1} \mathscr{E}_{0} \leqslant \mathscr{G}_{1}+\tau g_{0} \mathscr{E}_{1} \mathscr{G}_{1}, \quad \tau g_{0}<1 / \mathscr{G}_{1}
$$

Hence,

$$
\mathscr{E}_{1} \leqslant \frac{\mathscr{G}_{1}}{1-\mathscr{G}_{1} \tau g_{0}}
$$

Consequently, for $n=0$ estimate (2.17) holds. Now assume that inequality (2.17) holds for $n=p \geqslant 1$. Then, taking into account the properties of the function $\mathscr{G}_{n}$ and inequalities $(2.15)$ and (2.16), we have

$$
\mathscr{E}_{p+2} \leqslant \mathscr{G}_{p+2}+\sum_{k=0}^{p+1} \tau g_{k} \mathscr{E}_{k+1} \mathscr{E}_{k} \leqslant \mathscr{G}_{p+2}+\frac{\tau g_{p+1} \mathscr{E}_{p+2} \mathscr{G}_{p+1}+\mathscr{G}_{p+1} \mathscr{G}_{p} \sum_{k=0}^{p} \tau g_{k}}{1-\mathscr{G}_{p+1} \sum_{k=0}^{p} \tau g_{k}} .
$$

Hence,

$$
\left(1-\mathscr{G}_{p+1} \sum_{k=0}^{p+1} \tau g_{k}\right) \mathscr{E}_{p+2} \leqslant \mathscr{G}_{p+2}-\mathscr{G}_{p+1}\left(\mathscr{G}_{p+2}-\mathscr{G}_{p}\right) \sum_{k=0}^{p} \tau g_{k} .
$$

On the assumption of lemma $\mathscr{G}_{p+2} \geqslant \mathscr{G}_{p+1} \geqslant \mathscr{G}_{p}$. Thus from (2.18) it follows that inequality (2.17) also holds for $n=p+1$. This completes the proof.

Below Lemma 2.4 is used to obtain a priori estimates of the difference derivative of the solution of the implicit difference scheme approximating the nonlinear transport equation. 


\section{Difference schemes for the nonlinear scalar conservation law}

Let us study the properties of the difference algorithms approximating problems for nonlinear equations.

In the domain $\bar{Q}=\{(t, x): 0 \leqslant t \leqslant T, 0 \leqslant x \leqslant l\}$, consider the problem

$$
\begin{gathered}
\frac{\partial u}{\partial t}+u \frac{\partial u}{\partial x}=f(t, x), \quad 0<t \leqslant T, \quad 0<x \leqslant l, \\
u(t, 0)=\mu(t), \quad 0<t \leqslant T, \quad u(0, x)=u_{0}(x), \quad 0 \leqslant x \leqslant l .
\end{gathered}
$$

Suppose that the input data satisfy the following conditions:

$$
\begin{gathered}
u_{0}(x) \in C^{1}[0, l], \quad \mu(t) \in C^{1}[0, T] \\
\mu(t) \geqslant m_{1}>0, \quad u_{0}(x) \geqslant m_{2}>0, \quad f(t, x) \geqslant 0, \quad 0 \leqslant t \leqslant T, \quad 0 \leqslant x \leqslant l .
\end{gathered}
$$

In the domain $\bar{Q}$, let us introduce the grids

$$
\begin{gathered}
\bar{\omega}=\bar{\omega}_{h} \times \bar{\omega}_{\tau}, \quad \bar{\omega}_{h}=\left\{x_{i}=i h: i=0,1,2, \ldots, N_{x}\right\}=\omega_{h} \cup\left\{x_{0}=0\right\}, \\
\bar{\omega}_{\tau}=\left\{t_{n}=n \tau: n=0,1, \ldots, N\right\}=\omega_{\tau} \cup\{T=N \tau\} .
\end{gathered}
$$

3.1. Implicit difference scheme. On the grid $\bar{\omega}$ we approximate problem (3.1), (3.2) by the linearized implicit difference scheme

$$
\begin{gathered}
y_{t}+y \hat{y}_{\bar{x}}=\varphi, \quad \varphi(t, x)=f(t+\tau, x) \quad x \in \omega_{h}, \quad t \in \omega_{\tau}, \\
\hat{y}(t, 0)=\hat{\mu}(t), \quad t \in \omega_{\tau}, \quad y(0, x)=u_{0}, \quad x \in \bar{\omega}_{h} .
\end{gathered}
$$

Here we use the following notation of the theory of difference schemes [25]:

$$
y=y_{i}^{n}=y\left(t_{n}, x_{i}\right), \quad \hat{y}=y_{i}^{n+1}, \quad y_{t}=\frac{\hat{y}-y}{\tau}, \quad y_{\bar{x}}=\frac{y_{i}^{n}-y_{i-1}^{n}}{h} .
$$

Rewrite problem (3.4) in the form

$$
\begin{gathered}
\left(1+\gamma y_{i}^{n}\right) y_{i}^{n+1}=\gamma y_{i}^{n} y_{i-1}^{n+1}+y_{i}^{n}+\tau \varphi_{i}^{n}, \quad i=1,2, \ldots, N_{x}, \quad n=1,2, \ldots, N-1, \\
y_{0}^{n+1}=\mu^{n+1}, \quad n=0,1, \ldots, N-1, \quad y_{i}^{0}=u_{0, i}, \quad i=0,1, \ldots, N_{x}, \quad \gamma=\frac{\tau}{h} .
\end{gathered}
$$

Using Lemmas 2.1-2.3, conditions (3.3), and relations (3.5), it is easy to obtain (by induction on $n$ ) the estimate

$$
0<c_{1} \leqslant y_{i}^{n+1} \leqslant\left\|y^{n+1}\right\|_{C\left(\bar{\omega}_{h}\right)} \leqslant \max \left\{\max _{1 \leqslant k \leqslant n+1} \mu^{k},\left\|u_{0}\right\|_{C\left(\bar{\omega}_{h}\right)}\right\}+\sum_{k=0}^{N} \tau\left\|\varphi^{k}\right\|_{C\left(\omega_{h}\right)}=c_{2},
$$

where $c_{1}=\min \left\{m_{1}, m_{2}\right\}$.

Consider the difference scheme

$$
\begin{gathered}
\tilde{y}_{t}+\tilde{y} \hat{\tilde{y}}_{\bar{x}}=\tilde{\varphi}, \quad \tilde{\varphi}(t, x)=\tilde{f}(t+\tau, x), \quad x \in \omega_{h}, \quad t \in \omega_{\tau}, \\
\hat{\tilde{y}}(t, 0)=\tilde{\tilde{\mu}}(t), \quad t \in \omega_{\tau}, \quad \tilde{y}(0, x)=\tilde{u}_{0}, \quad x \in \bar{\omega}_{h},
\end{gathered}
$$


approximating the differential problem

$$
\begin{gathered}
\frac{\partial \tilde{u}}{\partial t}+\tilde{u} \frac{\partial \tilde{u}}{\partial x}=\tilde{f}(t, x), \quad 0<t \leqslant T, \quad 0<x \leqslant l, \\
\tilde{u}(t, 0)=\tilde{\mu}(t), \quad 0<t \leqslant T, \quad \tilde{u}(0, x)=\tilde{u}_{0}(x), \quad 0 \leqslant x \leqslant l,
\end{gathered}
$$

with perturbed input data

$$
\begin{gathered}
\tilde{u}_{0}(x) \in C^{1}[0, l], \quad \tilde{\mu}(t) \in C^{1}[0, T], \\
\tilde{\mu}(t) \geqslant \tilde{m}_{1}>0, \quad \tilde{u}_{0}(x) \geqslant \tilde{m}_{2}>0, \quad \tilde{f}(t, x) \geqslant 0, \quad 0 \leqslant t \leqslant T, \quad 0 \leqslant x \leqslant l,
\end{gathered}
$$

As above, for the solution $\tilde{y}$ of the difference scheme (3.7) the following estimate holds:

$$
0<\tilde{c}_{1} \leqslant \tilde{y}_{i}^{n+1} \leqslant\left\|\tilde{y}^{n+1}\right\|_{C\left(\bar{\omega}_{h}\right)} \leqslant \max \left\{\max _{1 \leqslant k \leqslant n+1} \tilde{\mu}^{k},\left\|\tilde{u}_{0}\right\|_{C\left(\bar{\omega}_{h}\right)}\right\}+\sum_{k=0}^{N} \tau\left\|\tilde{\varphi}^{k}\right\|_{C\left(\omega_{h}\right)}=\tilde{c}_{2},
$$

where $\tilde{c}_{1}=\min \left\{\tilde{m}_{1}, \tilde{m}_{2}\right\}$.

To investigate the stability and monotonicity of the difference scheme (3.4), we write the problem for perturbation $\delta y=\tilde{y}-y$

$$
\begin{gathered}
(\delta y)_{t}+y(\delta \hat{y})_{\bar{x}}+\delta y \hat{\tilde{y}}_{\bar{x}}=\delta \varphi, \quad x \in \omega_{h}, t \in \omega_{\tau}, \\
\delta y(t, 0)=\delta \mu(t), \quad t \in \omega_{\tau}, \quad \delta y(0, x)=\delta u_{0}, \quad x \in \bar{\omega}_{h} .
\end{gathered}
$$

Problem (3.12) can be rewritten in the following form:

$$
\begin{gathered}
\left(1+\gamma y_{i}^{n}\right) \delta y_{i}^{n+1}=\gamma y_{i}^{n} \delta y_{i-1}^{n+1}+\left(1-\tau \tilde{y}_{\bar{x}, i}^{n+1}\right) \delta y_{i}^{n}+\tau \delta \varphi_{i}^{n}, \quad i=1,2, \ldots, N_{x}, \quad n=0,1, \ldots, N-1, \\
\delta y_{0}^{n+1}=\delta \mu^{n+1}, \quad n=0,1, \ldots, N-1, \quad \delta y_{i}^{0}=\delta u_{0 i}, \quad i=0,1, \ldots, N_{x} .
\end{gathered}
$$

From the structure of (3.13) we see that for investigating the stability and monotonicity of the difference scheme (3.4) we need information about the first difference derivative $\tilde{y}_{\bar{x}, i}^{n+1}$. This is one of distinguishing features of investigating the stability and monotonicity in the nonlinear case.

Since the difference equation (3.4) must be satisfied at the grid point $x_{0}=0$, then, using the boundary conditions, we determine $\tilde{y}(t,-h)$ and $\tilde{u}_{0}(-h)$ as follows:

$$
\tilde{y}(t,-h)=\tilde{\mu}(t)+h \frac{\tilde{\mu}_{t}(t)-\tilde{\varphi}(t, 0)}{\tilde{\mu}(t)}, \quad \tilde{u}_{0}(-h)=\tilde{\mu}(0)+h \frac{\tilde{\mu}_{t}(0)-\tilde{\varphi}(0,0)}{\tilde{\mu}(0)} .
$$

Now by the difference equations (3.7) we obtain the problem for the grid function $w=\tilde{y}_{\bar{x}}$

$$
\begin{gathered}
w_{t}+(\tilde{y} \hat{w})_{\bar{x}}=\tilde{\varphi}_{\bar{x}}, \quad t \in \omega_{\tau}, \quad x \in \omega_{h}, \\
\hat{w}(t, 0)=\hat{\tilde{\mu}}_{1}(t)=\frac{\hat{\tilde{\varphi}}(t, 0)-\hat{\tilde{\mu}}_{t}(t)}{\hat{\tilde{\mu}}(t)}, \quad t \in \omega_{\tau}, \quad w(0, x)=\tilde{u}_{0 \bar{x}}(x), \quad x \in \bar{\omega}_{h} .
\end{gathered}
$$

Moreover, the boundary and initial conditions are consistent, i.e., $\tilde{u}_{0}(0)=\tilde{\mu}_{1}(0)$. The last problem can be rewritten in the following form:

$$
\begin{aligned}
\left(1+\gamma \tilde{y}_{i}^{n}\right) w_{i}^{n+1} & =\gamma \tilde{y}_{i-1}^{n} w_{i-1}^{n+1}+w_{i}^{n}+\tau \tilde{\varphi}_{\bar{x}, i}^{n}, \quad i=1,2, \ldots, N_{x}, \quad n=0,1, \ldots, N-1, \\
w_{0}^{n+1} & =\tilde{\mu}_{1}^{n+1}, \quad n=0,1, \ldots, N-1, \quad w_{i}^{0}=\tilde{u}_{0 \bar{x}, i}, \quad i=0,1, \ldots, N_{x} .
\end{aligned}
$$


3.1.1. Investigation of stability in the case where a shock wave is not generated. In this case, the input data of problem (3.1), (3.2) and the corresponding perturbed problem (3.8), (3.9) satisfy, in addition, the conditions

$$
u_{0}^{\prime}(x), \tilde{u}_{0}^{\prime}(x) \geqslant 0, \quad \frac{\partial f(t, x)}{\partial x}, \frac{\partial \tilde{f}(t, x)}{\partial x} \geqslant 0, \quad \mu^{\prime}(t), \tilde{\mu}^{\prime}(t) \leqslant 0, \quad(t, x) \in \bar{Q} .
$$

Under conditions (3.3), (3.10), (3.16) it can be proved by induction on $n$ that all coefficients of problem (3.14), (3.15) are positive and

$$
w \geqslant 0, \quad t \in \bar{\omega}_{\tau}, \quad x \in \omega_{h} .
$$

Now we obtain the estimate of the grid function $w$. Suppose that $w$ reaches its maximum at the grid point $x_{0}=0$; then $\left\|w^{n+1}\right\|_{C\left(\bar{\omega}_{h}\right)}=\mu_{1}^{n+1}$. If $w$ reaches its maximum at a certain grid point $x_{i^{*}}$ of the grid $\omega_{h}$, i.e., $\left\|w^{n+1}\right\|_{C\left(\bar{\omega}_{h}\right)}=w_{i^{*}}^{n+1}$; then from (3.14) we obtain

$$
\left(1+\tau w_{i^{*}}^{n}\right) w_{i^{*}}^{n+1}=w_{i^{*}}^{n}+\tau \tilde{\varphi}_{\bar{x}, i^{*}}^{n}
$$

Taking into account the nonnegativity of $w$, we get

$$
\left\|w^{n+1}\right\|_{C\left(\bar{\omega}_{h}\right)} \leqslant\left\|w^{n}\right\|_{C\left(\bar{\omega}_{h}\right)}+\tau\left\|\tilde{\varphi}_{\bar{x}}^{n}\right\|_{C\left(\omega_{h}\right)} .
$$

Hence, for all $x \in \bar{\omega}_{h}$ the following recurrent inequality holds:

$$
\left\|w^{n+1}\right\|_{C\left(\bar{\omega}_{h}\right)} \leqslant \max \left\{\mu_{1}^{n+1},\left\|w^{n}\right\|_{C\left(\bar{\omega}_{h}\right)}\right\}+\tau\left\|\tilde{\varphi}_{\bar{x}}^{n}\right\|_{C\left(\omega_{h}\right)},
$$

Using Lemma 2.3, from the last inequality we get the following estimate:

$$
\left\|w^{n+1}\right\|_{C\left(\bar{\omega}_{h}\right)} \leqslant \max \left\{\max _{1 \leqslant k \leqslant N} \mu_{1}^{k},\left\|w^{0}\right\|_{C\left(\bar{\omega}_{h}\right)}\right\}+\sum_{k=0}^{N} \tau\left\|\tilde{\varphi}_{\bar{x}}^{k}\right\|_{C\left(\omega_{h}\right)}=\tilde{c}_{3} .
$$

Now we estimate the perturbation $\delta y$. Since

$$
1-\tau \tilde{y}_{\bar{x}}^{n+1} \geqslant 1-\tau \tilde{c}_{3} \geqslant 0, \quad \text { if } \tau \leqslant \tau_{0}=1 / \tilde{c}_{3},
$$

it follows that the coefficients of problem (3.13) are positive for sufficiently small $\tau \leqslant \tau_{0}$. Using Lemma 2.2 and the nonnegativity of $\tilde{y}_{\bar{x}}$, for sufficiently small $\tau \leqslant \tau_{0}$, we obtain the inequality

$$
\begin{gathered}
\left\|\delta y^{n+1}\right\|_{C\left(\bar{\omega}_{h}\right)} \leqslant \\
\max \left\{\left|\delta \mu^{n+1}\right|,\left(1-\tau \tilde{y}_{\bar{x}, i^{*}}^{n+1}\right)\left\|\delta y^{n}\right\|_{C\left(\bar{\omega}_{h}\right)}\right\}+\tau\left\|\delta \varphi^{n}\right\|_{C\left(\omega_{h}\right)} \leqslant \\
\max \left\{\left|\delta \mu^{n+1}\right|,\left\|\delta y^{n}\right\|_{C\left(\bar{\omega}_{h}\right)}\right\}+\tau\left\|\delta \varphi^{n}\right\|_{C\left(\omega_{h}\right)} .
\end{gathered}
$$

Here $i^{*}$ is the number of the grid point where $\left\|\delta y^{n+1}\right\|_{C\left(\omega_{h}\right)}=\left|\delta y_{i^{*}}^{n+1}\right|$. Hence, using Lemma 2.3, we get the following estimate:

$$
\left\|\tilde{y}^{n+1}-y^{n+1}\right\|_{C\left(\bar{\omega}_{h}\right)} \leqslant \max \left\{\max _{1 \leqslant k \leqslant n+1}\left|\tilde{\mu}^{k}-\mu^{k}\right|,\left\|\tilde{u}_{0}-u_{0}\right\|_{C\left(\bar{\omega}_{h}\right)}\right\}+\sum_{k=0}^{n} \tau\left\|\tilde{\varphi}^{n}-\varphi^{n}\right\|_{C\left(\omega_{h}\right)},
$$

expressing the stability with respect to the input data. Moreover, the above conditions ensure the unconditional monotonicity of the difference scheme for sufficiently small $\tau \leqslant \tau_{0}$. 
3.1.2. Investigation of stability in the case where a shock wave is generated. Now let the following conditions hold:

$$
u_{0}^{\prime}(x), \tilde{u}_{0}^{\prime}(x) \leqslant 0, \quad \frac{\partial f(t, x)}{\partial x}, \frac{\partial \tilde{f}(t, x)}{\partial x} \leqslant 0, \quad \mu^{\prime}(t), \tilde{\mu}^{\prime}(t) \geqslant 0, \quad(t, x) \in \bar{Q},
$$

and the strict inequalities are satisfied at least at one point.

Let us obtain the estimate of $w$. Let the absolute value of the function $w_{i}^{n+1}$ reach its maximum at a certain grid point $x_{i^{*}}>0$ of the grid $\bar{\omega}_{h}$, i.e., $\left\|w^{n+1}\right\|_{C\left(\bar{\omega}_{h}\right)}=\left|w_{i^{*}}^{n+1}\right|$. Then from (3.14) it follows that

$$
\left(1+\tau w_{i^{*}}^{n}\right)\left\|w^{n+1}\right\|_{C\left(\bar{\omega}_{h}\right)} \leqslant\left\|w^{n}\right\|_{C\left(\bar{\omega}_{h}\right)}+\tau\left\|\tilde{\varphi}_{\bar{x}}^{n}\right\|_{C\left(\omega_{h}\right)}
$$

and, taking into account $1+\tau w_{i^{*}}^{n} \geqslant 1-\tau\left\|w^{n}\right\|_{C\left(\bar{\omega}_{h}\right)}$, we obtain the following nonlinear relation:

$$
\left(1-\tau\left\|w^{n}\right\|_{C\left(\bar{\omega}_{h}\right)}\right)\left\|w^{n+1}\right\|_{C\left(\bar{\omega}_{h}\right)} \leqslant\left\|w^{n}\right\|_{C\left(\bar{\omega}_{h}\right)}+\tau\left\|\tilde{\varphi}_{\bar{x}}^{n}\right\|_{C\left(\omega_{h}\right)} .
$$

If the absolute value of the grid function $w_{i}^{n+1}$ reaches its maximum at the grid point $x_{0}=0$, then $\left\|w^{n+1}\right\|_{C\left(\bar{\omega}_{h}\right)}=\left|\mu_{1}^{n+1}\right|$. Thus, we get the nonlinear recurrent inequality

$$
\left\|w^{n+1}\right\|_{C\left(\bar{\omega}_{h}\right)} \leqslant \max \left\{\left|\mu_{1}^{n+1}\right|,\left\|w^{n}\right\|_{C\left(\bar{\omega}_{h}\right)}\right\}+\tau\left\|w^{n}\right\|_{C\left(\bar{\omega}_{h}\right)}\left\|w^{n+1}\right\|_{C\left(\bar{\omega}_{h}\right)}+\tau\left\|\tilde{\varphi}_{\bar{x}}^{n}\right\|_{C\left(\omega_{h}\right)} .
$$

By analogy with the proof of Lemma 2.3, from the last inequality we get the relation

$$
\left\|w^{n+1}\right\|_{C\left(\bar{\omega}_{h}\right)} \leqslant \max \left\{\max _{1 \leqslant k \leqslant n}\left|\mu_{1}^{k+1}\right|,\left\|w^{0}\right\|_{C\left(\bar{\omega}_{h}\right)}\right\}+\sum_{k=0}^{n} \tau\left\|w^{k}\right\|_{C\left(\bar{\omega}_{h}\right)}\left\|w^{k+1}\right\|_{C\left(\bar{\omega}_{h}\right)}+\sum_{k=0}^{n} \tau\left\|\tilde{\varphi}_{\bar{x}}^{k}\right\|_{C\left(\omega_{h}\right)} .
$$

To obtain the estimate with respect to only the initial and boundary conditions and the right-hand side, we apply Lemma 2.4. Let

$$
\begin{gathered}
\mathscr{E}_{n}=\left\|w^{n}\right\|_{C\left(\bar{\omega}_{h}\right)}, \quad \mathscr{G}_{n+1}=\max \left\{\max _{1 \leqslant k \leqslant n}\left|\mu_{1}^{k+1}\right|,\left\|w^{0}\right\|_{C\left(\bar{\omega}_{h}\right)}\right\}+\sum_{k=0}^{n} \tau\left\|\tilde{\varphi}_{\bar{x}}^{k}\right\|_{C\left(\omega_{h}\right)}, \\
g_{n}=1, \quad \text { for all } n=0,1,2, \ldots, N .
\end{gathered}
$$

Then condition (2.16) yields

$$
\left(\max \left\{\max _{1 \leqslant k \leqslant n}\left|\mu_{1}^{k+1}\right|,\left\|w^{0}\right\|_{C\left(\bar{\omega}_{h}\right)}\right\}+\sum_{k=0}^{n} \tau\left\|\tilde{\varphi}_{\bar{x}}^{k}\right\|_{C\left(\omega_{h}\right)}\right) t_{n}<1 .
$$

If the stronger condition

$$
\left(\max \left\{\max _{1 \leqslant k \leqslant n}\left|\mu_{1}^{k+1}\right|,\left\|w^{0}\right\|_{C\left(\bar{\omega}_{h}\right)}\right\}+\sum_{k=0}^{n} \tau\left\|\tilde{\varphi}_{\bar{x}}^{k}\right\|_{C\left(\omega_{h}\right)}\right) t_{n}<1-\varepsilon, \quad 0<\varepsilon<1,
$$

holds, then for $\left\|w^{n+1}\right\|_{\bar{C}}$ the estimate

$$
\left\|w^{n+1}\right\|_{C\left(\bar{\omega}_{h}\right)} \leqslant \frac{1}{\varepsilon}\left(\max \left\{\max _{1 \leqslant k \leqslant n^{*}}\left|\mu_{1}^{k+1}\right|,\left\|w^{0}\right\|_{C\left(\bar{\omega}_{h}\right)}\right\}+\sum_{k=0}^{n^{*}} \tau\left\|\tilde{\varphi}_{\bar{x}}^{k}\right\|_{C\left(\omega_{h}\right)}\right)=\tilde{c}_{4}
$$

holds on the interval $\left[0, t_{n^{*}}\right]$, where $t_{n^{*}}$ is determined by inequality $(3.23)$ 
Now we obtain the estimate of the perturbation $\delta y$ of the difference solution. Taking into account inequalities (3.6), (3.11) and (3.24), from (3.13) we get

$$
\left\|\delta y^{n+1}\right\|_{C\left(\bar{\omega}_{h}\right)} \leqslant\left(1+\tilde{c}_{4} \tau\right) \max \left\{\left|\delta \mu^{n+1}\right|,\left\|\delta y^{n}\right\|_{C\left(\bar{\omega}_{h}\right)}\right\}+\tau\left\|\delta \varphi^{n}\right\|_{C\left(\omega_{h}\right)}, \quad n=0,1, \ldots n^{*}-1 .
$$

Therefore, using the discrete Gronwall inequality [25], we obtain the estimate

$$
\begin{gathered}
\left\|\tilde{y}^{n+1}-y^{n+1}\right\|_{C\left(\bar{\omega}_{h}\right)} \leqslant e^{\tilde{c}_{4} t_{n+1}} \max \left\{\max _{1 \leqslant k \leqslant n+1}\left|\tilde{\mu}^{k}-\mu^{k}\right|,\left\|\tilde{u}_{0}-u_{0}\right\|_{C\left(\bar{\omega}_{h}\right)}\right\}+ \\
\sum_{k=0}^{n} \tau e^{\tilde{c}_{4}\left(t_{n}-t_{k}\right)}\left\|\tilde{\varphi}^{n}-\varphi^{n}\right\|_{C\left(\omega_{h}\right)}, \quad n=0,1, \ldots, n^{*}-1,
\end{gathered}
$$

expressing the $\rho$-stability of scheme (3.4).

Thus we have shown that scheme $(3.4)$ is stable on the interval $\left[0, t_{n^{*}}\right]$, where $t_{n^{*}}$ is determined by inequality (3.23).

3.2. Conservative, exact and stable schemes on the shock wave. Consider the Riemann problem

$$
\begin{gathered}
\frac{\partial u}{\partial t}+\frac{\partial F(u)}{\partial x}=0, \quad 0<t \leqslant T, \quad 0<x \leqslant l, \quad F^{\prime}(u)>0, \quad F^{\prime \prime}(u)>0 \\
u(0, x)=u_{0}(x)=\left\{\begin{array}{ll}
u^{L} & \text { for } 0<x<\xi, \\
u^{R} & \text { for } \xi<x \leqslant l,
\end{array} u^{L}>u^{R} \geqslant 0 .\right. \\
u(t, 0)=u^{L}, \quad 0<t \leqslant T,
\end{gathered}
$$

where $u^{L}$ and $u^{R}$ are constants.

We are interested in the weak solution. The weak solution is defined in the class of piecewise continuous functions satisfying (for any closed piecewise-smooth contour $\partial V$ ) the following integral equation [9]

$$
\oint_{\partial V} u d x-F(u) d t=0
$$

and conditions (3.27) and (3.28).

Let the solution $u(t, x)$ be discontinuous along the line $x(t)=\xi+X(t)$. In the case of the considered Riemann problem (3.26)-(3.28), the velocity $D(t)$ of the discontinuity is determined by the Rankine-Hugoniot condition [8]:

$$
D=\frac{F\left(u^{R}\right)-F\left(u^{L}\right)}{u^{R}-u^{L}}=\frac{d X}{d t} .
$$

If $D \equiv$ const, then $D=d x / d t=h / \tau$. Thus, for the constant velocity $D$ it follows from (3.30) that

$$
\frac{F\left(u^{R}\right)-F\left(u^{L}\right)}{u^{R}-u^{L}}=\frac{h}{\tau} .
$$

Hence, assuming

$$
\frac{\tau D}{h}=1
$$


we obtain the identity

$$
\frac{u^{R}-u^{L}}{\tau}-\frac{F\left(u^{R}\right)-F\left(u^{L}\right)}{h}=0
$$

For $u^{R}$, from (3.32) we have

$$
u^{R}=u^{L}+\frac{\tau}{h}\left(F\left(u^{R}\right)-F\left(u^{L}\right)\right) .
$$

To approximate problem (3.26)-(3.28), we take the grid $\bar{\omega}_{h}$, as follows: the point $\xi$ (where the initial function $u_{0}$ is discontinuous) is not a grid-point. Let $\xi \in\left(x_{i_{0}-1}, x_{i_{0}}\right)$ (see figure).

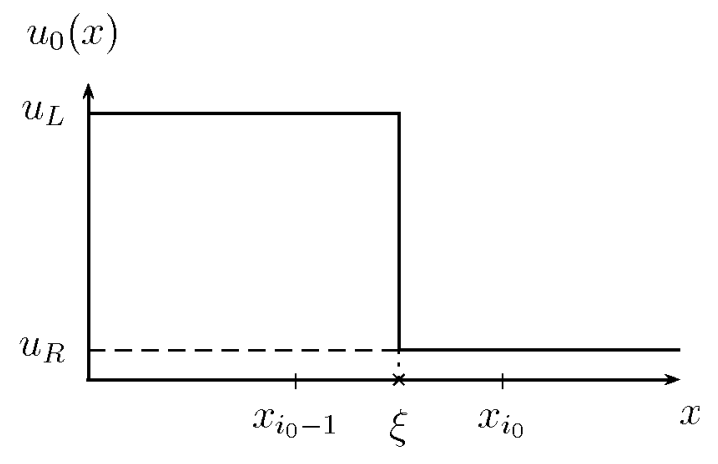

On the grid $\bar{\omega}$, consider the difference scheme

$$
\begin{gathered}
\frac{u_{i}^{n+1}-u_{i}^{n}}{\tau}+\frac{F\left(u_{i}^{n}\right)-F\left(u_{i-1}^{n}\right)}{h}=0, \quad t \in \omega_{\tau}, \quad x \in \omega_{h}, \\
u(0, x)=u_{0}(x), \quad x \in \bar{\omega}_{h}, \quad u(t, 0)=u^{L}, \quad t \in \omega_{\tau} .
\end{gathered}
$$

From equation (3.33) we have

$$
u_{i}^{n+1}=u_{i}^{n}-\frac{\tau}{h}\left(F\left(u_{i}^{n}\right)-F\left(u_{i-1}^{n}\right)\right) .
$$

Thus, since $u_{i_{0}-1}^{n}=u^{L}, u_{i_{0}}^{n}=u^{R}$ near the discontinuity, it follows that

$$
u_{i_{0}}^{n+1}=u^{R}-\frac{\tau}{h}\left(F\left(u^{R}\right)-F\left(u^{L}\right)\right) .
$$

Combining this with (3.32), we get the identity

$$
u_{i_{0}}^{n+1}=u^{L}=u_{i_{0}-1}^{n},
$$

and, consequently, the difference equation (3.33) approximates the Rankine-Hugoniot conditions (3.32) exactly near the discontinuity. It follows that on the shock wave under assumptions (3.31) the difference scheme (3.33) approximates problem (3.26)-(3.28) exactly.

Consider the problem with perturbed input data

$$
\begin{gathered}
\frac{\partial \tilde{u}}{\partial t}+\frac{\partial F(\tilde{u})}{\partial x}=0, \quad 0<t \leqslant T, \quad 0<x \leqslant l, \quad F^{\prime}(\tilde{u})>0, \quad F^{\prime \prime}(\tilde{u})>0 \\
\tilde{u}(0, x)=\tilde{u}_{0}(x)=\left\{\begin{array}{ll}
\tilde{u}^{L} & \text { for } 0<x<\xi, \\
\tilde{u}^{R} & \text { for } \xi<x \leqslant l,
\end{array} \tilde{u}^{L}>\tilde{u}^{R} \geqslant 0 .\right.
\end{gathered}
$$




$$
\tilde{u}(t, 0)=\tilde{u}^{L}, \quad 0<t \leqslant T,
$$

where $\tilde{u}^{L}$ and $\tilde{u}^{R}$ are constants. On the grid $\bar{\omega}$ we approximate this problem by the following difference scheme:

$$
\begin{gathered}
\frac{\tilde{u}_{i}^{n+1}-\tilde{u}_{i}^{n}}{\tau}+\frac{F\left(\tilde{u}_{i}^{n}\right)-F\left(\tilde{u}_{i-1}^{n}\right)}{h}=0, \quad t \in \omega_{\tau}, \quad x \in \omega_{h}, \\
\tilde{u}(0, x)=\tilde{u}_{0}(x), \quad x \in \bar{\omega}_{h}, \quad \tilde{u}(t, 0)=\tilde{u}^{L}, \quad t \in \omega_{\tau} .
\end{gathered}
$$

The difference scheme (3.38), (3.39) approximates problem (3.35), (3.36) exactly on the same grid $\bar{\omega}$ with

$$
\tilde{D}=\frac{F\left(\tilde{u}^{R}\right)-F\left(\tilde{u}^{L}\right)}{\tilde{u}^{R}-\tilde{u}^{L}}=\frac{h}{\tau}=D .
$$

Under conditions (3.31), (3.40) we have

$$
\begin{aligned}
& u_{i}^{n+1}=u_{i-1}^{n}, \quad i=1,2, \ldots, N_{x}, \quad n=0,1, \ldots, N-1, \\
& \tilde{u}_{i}^{n+1}=\tilde{u}_{i-1}^{n}, \quad i=1,2, \ldots, N_{x}, \quad n=0,1, \ldots, N-1 .
\end{aligned}
$$

Therefore,

$$
\delta u_{i}^{n+1}=\delta u_{i-1}^{n}, \quad i=1,2, \ldots, N_{x}, \quad n=0,1, \ldots, N-1
$$

Consequently,

$$
\left\|\delta u^{n+1}\right\|_{C\left(\bar{\omega}_{h}\right)} \leqslant\left\|\delta u_{0}\right\|_{C\left(\bar{\omega}_{h}\right)} .
$$

Thus, we have proved that the simple explicit conservative difference scheme (3.33), (3.34), approximating the Riemann problem (3.26) - (3.28), is stable and exact for $\tau=h D>\tau_{K}$ and $\tilde{D}=D$. We stress that this value of $\tau$ is greater than the value $\tau_{K}$ admissible by the Courant-Friedrichs-Lewy condition.

3.3. Difference schemes for multidimensional problems. In the domain $\bar{Q}^{d+1}=$ $\bar{\Omega}^{d} \times[0, T], \bar{\Omega}^{d}=\left\{\mathbf{x}=\left(x_{1}, x_{2}, \ldots, x_{d}\right) \in \mathbb{R}^{d}: 0 \leqslant x_{k} \leqslant l_{k}, k=1,2, \ldots, d\right\}=\Omega^{d} \cup \partial \Omega^{d}, \partial \Omega^{d}=$ $\left\{\mathbf{x} \in \bar{\Omega}^{d}: x_{k}=0, k=1,2, \ldots, d\right\}$, consider the multi-dimensional scalar conservation law

$$
\begin{gathered}
\frac{\partial u}{\partial t}+\sum_{k=1}^{d} \alpha_{k} \frac{\partial\left(u^{2}\right)}{\partial x_{k}}=0, \quad(t, \mathbf{x}) \in Q^{d+1}=\Omega^{d} \times(0, T], \\
\left.u(t, \mathbf{x})\right|_{\partial \Omega^{d}}=\mu(t, \mathbf{x}), \quad t \in(0, T] \quad u(0, \mathbf{x})=u_{0}(\mathbf{x}), \quad \mathbf{x} \in \bar{\Omega}^{d} .
\end{gathered}
$$

Suppose that $\alpha_{k}=$ const $>0$ and the functions defining the input data satisfy the following conditions:

$$
\begin{gathered}
0<m_{1} \leqslant u_{0}(\mathbf{x}) \leqslant m_{1}^{*}, \quad \mu(t, \mathbf{x}) \geqslant m_{2}>0, \quad \sum_{k=1}^{d} \alpha_{k} \frac{\partial u_{0}^{2}}{\partial x_{k}} \geqslant m_{3}>0, \quad \frac{\partial \mu}{\partial t} \leqslant 0, \\
u_{0}(\mathbf{x})=\mu(0, \mathbf{x}), \quad \sum_{k=1}^{d} \alpha_{k} \frac{\partial u_{0}(\mathbf{x})}{\partial x_{k}}=-\frac{\partial \mu(0, \mathbf{x})}{\partial t} \mu^{-1}(0, \mathbf{x}) \quad \mathbf{x} \in \partial \Omega^{d}, \\
\mu(t, \mathbf{x}) \in C^{1}\left([0, T] \times \partial \Omega^{d}\right), \quad u_{0}(\mathbf{x}) \in C^{1}\left(\bar{\Omega}^{d}\right) .
\end{gathered}
$$

Under conditions (3.43) the shock wave is not generated because in this case we have

$$
0<m_{3} \leqslant \sum_{k=1}^{d} \alpha_{k} \frac{\partial u_{0}^{2}}{\partial x_{k}}=2 u_{0} \sum_{k=1}^{d} \alpha_{k} \frac{\partial u_{0}}{\partial x_{k}} \leqslant 2 m_{1}^{*} \sum_{k=1}^{d} \alpha_{k} \frac{\partial u_{0}}{\partial x_{k}}
$$


and, consequently,

$$
\sum_{k=1}^{d} \alpha_{k} \frac{\partial u_{0}}{\partial x_{k}} \geqslant m_{4}=\frac{m_{3}}{2 m_{1}^{*}}>0 .
$$

In $\bar{Q}^{d+1}$, let us introduce the uniform grids

$$
\begin{gathered}
\bar{\omega}_{h}^{d}=\left\{\mathbf{x} \in \bar{\Omega}^{d}: x_{k}=i_{k} h_{k}, i_{k}=0,1, \ldots, N_{x_{k}}, h_{k} N_{x_{k}}=l_{k}, k=1,2, \ldots, d\right\}=\omega_{h}^{d} \cup \partial \omega_{h}^{d}, \\
\omega_{h}^{d}=\bar{\omega}_{h}^{d} \cap \Omega^{d}, \quad \partial \omega_{h}^{d}=\bar{\omega}_{h}^{d} \cap \partial \Omega^{d}, \quad \bar{\omega}^{d+1}=\bar{\omega}_{h}^{d} \times \bar{\omega}_{\tau}, \\
\partial \omega^{d+1}=\partial \omega_{h}^{d} \times \omega_{\tau} \cup\left\{(0, \mathbf{x}): \mathbf{x} \in \bar{\omega}_{h}^{d}\right\}, \quad h=\max \left(h_{1}, h_{2}, \ldots, h_{d}\right) .
\end{gathered}
$$

On the grid $\bar{\omega}^{d+1}$ we approximate problem (3.41), (3.42) by the following explicit conservative difference scheme:

$$
\begin{gathered}
y_{t}+\sum_{k=1}^{d} \alpha_{k}\left(y^{2}\right)_{\bar{x}_{k}}=0, \quad(t, \mathbf{x}) \in \omega^{d+1}=\omega_{h}^{d} \cup \omega_{\tau}, \\
\left.y\right|_{\partial \omega_{h}^{d}}=\mu(t, \mathbf{x}), \quad t \in \omega_{\tau}, \quad y(0, \mathbf{x})=u_{0}(\mathbf{x}), \quad \mathbf{x} \in \bar{\omega}_{h}^{d} .
\end{gathered}
$$

Here and below we use the following notation:

$$
\begin{gathered}
y=y(t, \mathbf{x}), \quad \hat{y}=y(t+\tau, \mathbf{x}), \quad \check{y}=y(t-\tau, \mathbf{x}), \\
y_{\left( \pm 1_{k}\right)}=y\left(t, \mathbf{x}_{\left( \pm 1_{k}\right)}\right)=y\left(t, x_{1}, \ldots, x_{k} \pm h_{k}, \ldots, x_{d}\right), \quad(t, \mathbf{x}) \in \bar{\omega}^{d+1}, \\
y^{(0.5)}=0.5(y+\check{y}), \quad y_{t}=\frac{\hat{y}-y}{\tau}, \quad y_{\bar{t}}=\frac{y-\check{y}}{\tau}, \quad y_{\bar{x}_{k}}=\frac{y-y_{\left(-i_{k}\right)}}{h_{k}} .
\end{gathered}
$$

Moreover, by definition, put

$$
\|v\|_{C\left(\bar{\omega}_{h}^{d}\right)}=\max _{\mathbf{x} \in \bar{\omega}_{h}^{d}}|v(\mathbf{x})|, \quad\|v\|_{C\left(\omega_{h}^{d}\right)}=\max _{\mathbf{x} \in \omega_{h}^{d}}|v(\mathbf{x})|, \quad\|v\|_{C\left(\partial \omega^{d+1}\right)}=\max _{\mathbf{x} \in \partial \omega^{d+1}}|v(\mathbf{x})| .
$$

The discrete analogues of condition (3.43) are needed for the sequel. Using expansion in Taylor series, we get

$$
\sum_{k=1}^{d} \alpha_{k}\left(u_{0}^{2}\right)_{\bar{x}_{k}}=\sum_{k=1}^{d} \alpha_{k} \frac{\partial u_{0}^{2}}{\partial x_{k}^{2}}-\sum_{k=1}^{d} \alpha_{k} \frac{h_{k}}{2} \frac{\partial^{2} \bar{u}_{k}^{2}}{\partial x_{k}^{2}} \geqslant m_{3}-h m_{5} \geqslant 0, \quad \text { for } h \leqslant h^{(1)}=\frac{m_{3}}{m_{5}}
$$

where

$$
m_{5}=\frac{1}{2} \max _{\mathbf{x} \in \bar{\Omega}^{d}}\left|\sum_{k=1}^{d} \alpha_{k} \frac{\partial^{2} u_{0}^{2}}{\partial x_{k}^{2}}\right|, \quad \bar{u}_{k}=u_{0}\left(x_{1}, \ldots, \bar{x}_{k}, \ldots, x_{d}\right), \quad \bar{x}_{k} \in\left(x_{k}-h_{k}, x_{k}+h_{k}\right) .
$$

In the same way, it can be proved that

$$
\sum_{k=1}^{d} \alpha_{k} u_{0 \bar{x}_{k}} \geqslant 0 \quad \text { for } h \leqslant h^{(2)}=\frac{m_{4}}{m_{6}}, \quad m_{6}=\frac{1}{2} \max _{\mathbf{x} \in \bar{\Omega}^{d}}\left|\sum_{k=1}^{d} \alpha_{k} \frac{\partial^{2} u_{0}}{\partial x_{k}^{2}}\right|
$$

Let us study the properties of the solution of the difference problem (3.46), (3.47). From equation (3.46) we obtain the solution on the upper level $\hat{y}$ as follows

$$
\hat{y}=\left(1-\tau y \sum_{k=1}^{d} \frac{\alpha_{k}}{h_{k}}\right) y+\tau \sum_{k=1}^{d} \frac{\alpha_{k}}{h_{k}} y_{\left(-1_{k}\right)}^{2}, \quad t \in \omega_{\tau}, \quad \mathbf{x} \in \omega_{h}^{d} .
$$


Lemma 3.1. Let on the $n$th time level $t=t_{n}$ the following conditions be fulfilled:

$$
\begin{gathered}
0 \leqslant y(t, \mathbf{x}) \leqslant c_{2}, \quad \sum_{k=1}^{d} \alpha_{k}\left(y^{2}\right)_{\bar{x}_{k}}(t, \mathbf{x}) \geqslant 0, \quad \mathbf{x} \in \bar{\omega}_{h}, \quad c_{2}=\max \left\{\left\|u_{0}\right\|_{C\left(\bar{\omega}_{h}^{d}\right)},\|\mu\|_{C\left(\partial \omega_{h}^{d}\right)}\right\}, \\
1-2 \tau c_{2} \sum_{k=1}^{d} \frac{\alpha_{k}}{h_{k}} \geqslant 0, \quad h \leqslant h^{(0)}=\min \left\{h^{(1)}, h^{(2)}\right\} .
\end{gathered}
$$

Then on the next time level $t+\tau$ the following inequalities hold:

$$
0 \leqslant y(t+\tau, \mathbf{x}) \leqslant c_{2}, \quad \sum_{k=1}^{d} \alpha_{k}\left(y^{2}\right)_{\bar{x}_{k}}(t+\tau, \mathbf{x}) \geqslant 0, \quad \mathbf{x} \in \bar{\omega}_{h} .
$$

Proof. From (3.43) we see that under conditions (3.52) inequalities (3.51) hold true on the initial time level $t=0$. Now assume that inequalities (3.51) are fulfilled at any instant of time $t$. Then from (3.50) it follows that $y(t+\tau, \mathbf{x}) \geqslant 0, \mathbf{x} \in \bar{\omega}_{h}$.

To obtain the upper estimate

$$
\|y(t+\tau)\|_{C\left(\bar{\omega}_{h}^{d}\right)} \leqslant c_{2}
$$

we use the maximum principle technique. The absolute value of the function $\hat{y}$ can reach its maximum either on the boundary $\partial \omega_{h}^{d}$, i.e.,

$$
\|\hat{y}\|_{C\left(\bar{\omega}_{h}^{d}\right)}=\|\mu\|_{C\left(\partial \omega_{h}^{d}\right)},
$$

or at a certain grid point $\mathbf{x}^{*} \in \omega_{h}^{d}$, i.e. $\|\hat{y}\|_{C\left(\bar{\omega}_{h}^{d}\right)}=\hat{y}\left(\mathbf{x}^{*}\right)$. So, from (3.50), we obtain

$$
\begin{gathered}
\|\hat{y}\|_{C\left(\bar{\omega}_{h}^{d}\right)} \leqslant\left(1-\tau y\left(\mathbf{x}^{*}\right) \sum_{k=1}^{d} \frac{\alpha_{k}}{h_{k}}\right)\|y\|_{C\left(\bar{\omega}_{h}^{d}\right)}+\tau \sum_{k=1}^{d} \frac{\alpha_{k}}{h_{k}} y_{\left(-1_{k}\right)}\left(\mathbf{x}^{*}\right)\|y\|_{C\left(\bar{\omega}_{h}^{d}\right)} \leqslant \\
\left(1-\tau \sum_{k=1}^{d} \alpha_{k} y_{\bar{x}_{k}}\left(\mathbf{x}^{*}\right)\right)\|y\|_{C\left(\bar{\omega}_{h}^{d}\right)} .
\end{gathered}
$$

By the induction hypothesis,

$$
0 \leqslant \sum_{k=1}^{d} \alpha_{k}\left(y^{2}\right)_{\bar{x}_{k}}(t, \mathbf{x})=y(t, \mathbf{x}) \sum_{k=1}^{d} \alpha_{k} y_{\bar{x}_{k}}(t, \mathbf{x})-\sum_{k=1}^{d} \alpha_{k} h_{k}\left(y_{\bar{x}_{k}}(t, \mathbf{x})\right)^{2} \leqslant y(t, \mathbf{x}) \sum_{k=1}^{d} \alpha_{k} y_{\bar{x}_{k}}(t, \mathbf{x}) .
$$

Therefore, since $\sum_{k=1}^{d} \alpha_{k} y_{\bar{x}_{k}}(t, \mathbf{x}) \geqslant 0$ it follows from (3.56) that

$$
\|y(t+\tau)\|_{C\left(\bar{\omega}_{h}^{d}\right)} \leqslant\|y(t)\|_{C\left(\bar{\omega}_{h}^{d}\right)} .
$$

Combining (3.55) and (3.57), we get estimate (3.54).

Now we must prove that $\sum_{k=1}^{d} \alpha_{k} y_{\bar{x}_{k}}(t+\tau, \mathbf{x}) \geqslant 0, \mathbf{x} \in \omega_{h}^{d}$. Subtracting equation (3.46) at the instant of time $t$ from the same equation at the instant of time $t+\tau$ and denoting $y_{t}(t+\tau, \mathbf{x})$ by $v=v(t, \mathbf{x})$, we obtain

$$
v+2 \sum_{k=1}^{d} \alpha_{k} \tau\left(y^{(0.5)} \check{v}\right)_{\bar{x}_{k}}=\check{v}, \quad(t, \mathbf{x}) \in \omega^{d},
$$


or

$$
v=\left(1-2 \tau y^{(0.5)} \sum_{k=1}^{d} \frac{\alpha_{k}}{h_{k}}\right) \check{v}+2 \tau \sum_{k=1}^{d} \frac{\alpha_{k}}{h_{k}} y_{\left(-1_{k}\right)}^{(0.5)} \check{v}_{\left(-1_{k}\right)} .
$$

For the boundary conditions we have

$$
\left.v\right|_{\partial \omega_{h}^{d}}=\mu_{t}, \quad t \in \omega_{\tau}
$$

The initial conditions for the function $v$ can be obtained from equation (3.46) at the time $t=0$ and from the initial condition (3.47):

$$
v(0, \mathbf{x})=v_{0}(\mathbf{x})=-\sum_{k=1}^{d} \alpha_{k}\left(u_{0}^{2}\right)_{\bar{x}_{k}}, \quad \mathbf{x} \in \bar{\omega}_{h}^{d}
$$

By the inductive assumption,

$$
\check{v}=-\sum_{k=1}^{d} \alpha_{k}\left(y^{2}\right)_{\bar{x}_{k}} \leqslant 0 .
$$

Therefore, using the already proved estimate (3.54) and conditions (3.52), we have

$$
1-2 \tau y^{(0.5)} \sum_{k=1}^{d} \frac{\alpha_{k}}{h_{k}} \geqslant 1-2 \tau c_{2} \sum_{k=1}^{d} \frac{\alpha_{k}}{h_{k}} \geqslant 0
$$

Moreover, $y(t, \mathbf{x}), y(t+\tau, \mathbf{x}) \geqslant 0$ for all $\mathbf{x} \in \bar{\omega}_{h}$. Thus, from equation (3.59) it follows that $y_{t}(t+\tau, \mathbf{x}) \leqslant 0$ for all $\mathbf{x} \in \bar{\omega}_{h}$. Using equation (3.46) at $t+\tau$ by direct calculation we have $\sum_{k=1}^{d} \alpha_{k}\left(y^{2}\right)_{\bar{x}_{k}}(t+\tau, \mathbf{x}) \leqslant 0$ for all $\mathbf{x} \in \bar{\omega}_{h}$.

To investigate the stability, we consider the differential problem

$$
\begin{gathered}
\frac{\partial \tilde{u}}{\partial t}+\sum_{k=1}^{d} \alpha_{k} \frac{\partial\left(\tilde{u}^{2}\right)}{\partial x_{k}}=0, \quad(t, \mathbf{x}) \in Q^{d+1}=\Omega^{d} \times(0, T], \\
\left.\tilde{u}(t, \mathbf{x})\right|_{\partial \Omega^{d}}=\tilde{\mu}(t, \mathbf{x}), \quad \tilde{u}(0, \mathbf{x})=\tilde{u}_{0}(\mathbf{x}), \quad t>0, \quad \mathbf{x} \in \bar{\Omega}^{d},
\end{gathered}
$$

with perturbed input data

$$
0<\tilde{m}_{1} \leqslant \tilde{u}_{0}(\mathbf{x}) \leqslant \tilde{m}_{1}^{*}, \quad \tilde{\mu}(t, \mathbf{x}) \geqslant \tilde{m}_{2}>0, \quad \sum_{k=1}^{d} \alpha_{k} \frac{\partial \tilde{u}_{0}^{2}}{\partial x_{k}} \geqslant \tilde{m}_{3}>0, \quad \frac{\partial \tilde{\mu}}{\partial t} \leqslant 0 .
$$

As above, we also have

$$
\sum_{k=1}^{d} \alpha_{k} \frac{\partial \tilde{u}_{0}}{\partial x_{k}} \geqslant \tilde{m}_{4}=\frac{\tilde{m}_{3}}{2 \tilde{m}_{1}^{*}}>0 .
$$

In addition, suppose that

$$
\begin{gathered}
\tilde{u}_{0}(\mathbf{x})=\tilde{\mu}(0, \mathbf{x}), \quad \sum_{k=1}^{2} \alpha_{k} \frac{\partial \tilde{u}_{0}(\mathbf{x})}{\partial x_{k}}=-\frac{\partial \tilde{\mu}(0, \mathbf{x})}{\partial t} \tilde{\mu}^{-1}(0, \mathbf{x}) \quad \mathbf{x} \in \bar{\Omega}^{d}, \\
\tilde{\mu}(t, \mathbf{x}) \in C^{1}\left([0, T] \times \partial \Omega^{d}\right), \quad \tilde{u}_{0}(\mathbf{x}) \in C^{1}\left(\bar{\Omega}^{d}\right) .
\end{gathered}
$$


We approximate problem (3.62), (3.63) by the explicit difference scheme

$$
\begin{gathered}
\tilde{y}_{t}+\sum_{k=1}^{d} \alpha_{k}\left(\tilde{y}^{2}\right)_{\bar{x}_{k}}=0, \quad(t, \mathbf{x}) \in \omega^{d+1}=\omega_{h}^{d} \cup \omega_{\tau}, \\
\left.\tilde{y}\right|_{\partial \omega_{h}^{d}}=\tilde{\mu}(t, \mathbf{x}), \quad \tilde{y}(0, \mathbf{x})=\tilde{u}_{0}(\mathbf{x}), \quad t \in \omega_{\tau}, \quad \mathbf{x} \in \bar{\omega}_{h}^{d} .
\end{gathered}
$$

As for the unperturbed problem, it can be shown that

$$
\sum_{k=1}^{d} \alpha_{k}\left(\tilde{u}_{0}^{2}\right)_{\bar{x}_{k}} \geqslant 0 \quad \text { for } h \leqslant \tilde{h}^{(1)}=\frac{\tilde{m}_{3}}{\tilde{m}_{5}}, \quad \tilde{m}_{5}=\frac{1}{2} \max _{\mathbf{x} \in \bar{\Omega}^{d}}\left|\sum_{k=1}^{d} \alpha_{k} \frac{\partial^{2} \tilde{u}_{0}^{2}}{\partial x_{k}^{2}}\right|
$$

and

$$
\sum_{k=1}^{d} \alpha_{k} \tilde{u}_{0 \bar{x}_{k}} \geqslant 0 \quad \text { for } h \leqslant \tilde{h}^{(2)}=\frac{\tilde{m}_{4}}{\tilde{m}_{6}}, \quad \tilde{m}_{6}=\frac{1}{2} \max _{\mathbf{x} \in \bar{\Omega}^{d}}\left|\sum_{k=1}^{d} \alpha_{k} \frac{\partial^{2} \tilde{u}_{0}}{\partial x_{k}^{2}}\right|
$$

Similarly to Lemma 3.1 the following statement can be proved.

Lemma 3.2. Let on the $n$th time-level $t=t_{n}$ the following conditions be fulfilled:

$$
\begin{aligned}
0 \leqslant \tilde{y}(t, \mathbf{x}) \leqslant \tilde{c}_{2}, & \sum_{k=1}^{d} \alpha_{k}\left(\tilde{y}^{2}\right)_{\bar{x}_{k}}(t, \mathbf{x}) \geqslant 0, \quad \mathbf{x} \in \bar{\omega}_{h}, \quad \tilde{c}_{2}=\max \left\{\left\|\tilde{u}_{0}\right\|_{C\left(\bar{\omega}_{h}^{d}\right)},\|\tilde{\mu}\|_{C\left(\partial \omega_{h}^{d}\right)}\right\} \\
& 1-2 \tau \tilde{c}_{2} \sum_{k=1}^{d} \frac{\alpha_{k}}{h_{k}} \geqslant 0, \quad h \leqslant \tilde{h}^{(0)}=\min \left\{\tilde{h}^{(1)}, \tilde{h}^{(2)}\right\} .
\end{aligned}
$$

Then on the next time-level $t+\tau$ the following inequalities hold:

$$
0 \leqslant \tilde{y}(t+\tau, \mathbf{x}) \leqslant \tilde{c}_{2}, \quad \sum_{k=1}^{d} \alpha_{k}\left(\tilde{y}^{2}\right)_{\bar{x}_{k}}(t+\tau, \mathbf{x}) \geqslant 0, \quad \mathbf{x} \in \bar{\omega}_{h} .
$$

To investigate the stability of the considered difference scheme, we obtain the problem for the perturbation $\delta y=\tilde{y}-y$ :

$$
\begin{gathered}
(\delta y)_{t}+\sum_{k=1}^{d} \alpha_{k}((\tilde{y}+y) \delta y)_{\bar{x}_{k}}=0, \quad(t, \mathbf{x}) \in \omega^{d+1}, \\
\left.\delta y\right|_{\partial \omega_{h}^{d}}=0, \quad t \in \omega_{\tau}, \quad \delta y(0, \mathbf{x})=\delta u_{0}(\mathbf{x}), \quad \mathbf{x} \in \bar{\omega}_{h}^{d} .
\end{gathered}
$$

Expressing $\delta \hat{y}=\hat{\tilde{y}}-\hat{y}$ from equation (3.74), we get

$$
\delta \hat{y}=\left(1-\tau(\tilde{y}+y) \sum_{k=1}^{d} \frac{\alpha_{k}}{h_{k}}\right) \delta y+\tau \sum_{k=1}^{d} \frac{\alpha_{k}}{h_{k}}\left(\tilde{y}_{\left(-1_{k}\right)}+y_{\left(-1_{k}\right)}\right) \delta y_{\left(-1_{k}\right)}
$$

Now we prove the following

Theorem 3.1. Let the conditions of Lemmas 3.1, 3.2 hold and

$$
\tau \leqslant \frac{h}{2 c \sum_{k=1}^{d} \alpha_{k}}, \quad c=\max \left\{c_{2}, \tilde{c}_{2}\right\}, \quad h \leqslant h_{0}=\min \left\{h^{(0)}, \tilde{h}^{(0)}\right\} .
$$


Then the difference scheme (3.46), (3.47) is stable with respect to small perturbations of the initial and boundary conditions and for any $t \in \omega_{\tau}$ the following estimate holds:

$$
\|\tilde{y}(t)-y(t)\|_{C\left(\bar{\omega}_{h}\right)} \leqslant \max \left\{\max _{t \in \omega_{\tau}}\|\tilde{\mu}(t)-\mu(t)\|_{C\left(\partial \omega_{h}^{d}\right)},\left\|\tilde{u}_{0}-u_{0}\right\|_{C\left(\bar{\omega}_{h}^{d}\right)}\right\} .
$$

Proof. Since all coefficients on the right-hand side of equation (3.76) are positive, it follows that the difference scheme is monotone. Moreover, using the maximum principle technique, it can be shown that

$$
\|\delta y(t)\|_{C\left(\bar{\omega}_{h}^{d}\right)} \leqslant \max \left\{\|\mu(t)\|_{C\left(\partial \omega_{h}^{d}\right)}\left(1-\tau \sum_{k=1}^{d} \alpha_{k}\left(\tilde{y}\left(t, \mathbf{x}^{*}\right)+y\left(t, \mathbf{x}^{*}\right)\right)_{\bar{x}_{k}}\right)\|\delta y(t-\tau)\|_{C\left(\bar{\omega}_{h}^{d}\right)}\right\} .
$$

Taking into account conditions (3.77) and Lemmas 3.1, 3.2, we have

$$
1-\tau \sum_{k=1}^{d} \alpha_{k}\left(\tilde{y}\left(t, \mathbf{x}^{*}\right)+y\left(t, \mathbf{x}^{*}\right)\right)_{\bar{x}_{k}} \geqslant 0, \quad \sum_{k=1}^{d} \alpha_{k} y_{\bar{x}_{k}}\left(t-\tau, \mathbf{x}^{*}\right), \quad \sum_{k=1}^{d} \alpha_{k} \tilde{y}_{\bar{x}_{k}}\left(t-\tau, \mathbf{x}^{*}\right) \leqslant 0 .
$$

Consequently, from (3.79) we obtain the required estimate (3.78) expressing the stability of the difference scheme (3.46), (3.47) with respect to small perturbations of the initial and boundary conditions.

\section{Difference schemes for parabolic equations}

In this section, we study the stability and monotonicity of the explicit difference scheme approximating the initial-boundary value problem for the porous media equation given in the domain $\bar{Q}^{d+1}=\bar{\Omega}^{d} \times[0, T], \bar{\Omega}^{d}=\left\{\mathbf{x}=\left(x_{1}, x_{2}, \ldots, x_{d}\right) \in \mathbb{R}^{d}: 0 \leqslant x_{k} \leqslant l_{k}, k=\right.$ $1,2, \ldots, d\}=\Omega^{d} \cup \partial \Omega^{d}, \Omega^{d}=\left\{\mathbf{x} \in \mathbb{R}^{d}: 0<x_{k}<l_{k}, k=1,2, \ldots, d\right\}:$

$$
\begin{aligned}
\frac{\partial u}{\partial t} & =\Delta u^{m}+f, \quad 0<t \leqslant T, \quad \mathbf{x} \in \Omega^{d}, \quad m>1, \\
\left.u(t, \mathbf{x})\right|_{\partial \Omega^{d}} & =\mu(t, \mathbf{x}), \quad 0<t \leqslant T, \quad u(0, \mathbf{x})=u_{0}(\mathbf{x}), \quad \mathbf{x} \in \bar{\Omega}^{d} .
\end{aligned}
$$

Here $\Delta$ is the Laplacian $\Delta u=\sum_{k=1}^{d} \frac{\partial^{2} u}{\partial x_{k}^{2}}$.

Suppose that the following conditions are satisfied:

$\left.1^{\circ}\right) 0<\kappa_{1} \leqslant u_{0}(\mathbf{x}) \leqslant \kappa_{2}, \mathbf{x} \in \bar{\Omega}^{d}, u_{0}(\mathbf{x}) \in C^{4}\left(\bar{\Omega}^{d}\right), \mu(t) \in C^{2}(0, T]$;

$\left.2^{\circ}\right) \partial \mu(t, \mathbf{x}) / \partial t \leqslant 0, \quad f(t, \mathbf{x}) \geqslant 0, \partial f(t, \mathbf{x}) / \partial t \leqslant 0,0<t \leqslant T, \mathbf{x} \in \bar{\Omega}^{d} ;$

$\left.3^{\circ}\right) \Delta u_{0}^{m}+f(0, \mathbf{x}) \leqslant-\varepsilon, \varepsilon=$ const $>0$.

From $\left.1^{\circ}\right)-3^{\circ}$ ), in particular, it follows that

$$
\Delta u_{0}(\mathbf{x}) \leqslant-\varepsilon_{1}, \quad \varepsilon_{1}=\frac{\varepsilon}{m \kappa_{1}^{m-1}}>0 .
$$

Indeed, by direct calculation we get

$$
-\varepsilon \geqslant \Delta u_{0}^{m}+f(0, \mathbf{x}) \geqslant m u_{0}^{m-1} \Delta u_{0}+f(0, \mathbf{x}) \geqslant m u_{0}^{m-1} \Delta u_{0} \geqslant m \kappa_{1}^{m-1} \Delta u_{0} .
$$

4.1. Difference scheme. Without loss of generality it can be assumed that $m=2$. On the grid

$$
\bar{\omega}^{d+1}=\bar{\omega}_{h}^{d} \times \bar{\omega}_{\tau},
$$




$$
\begin{gathered}
\bar{\omega}_{h}^{d}=\left\{\mathbf{x} \in \bar{\Omega}^{d}: x_{k}=i_{k} h_{k}, i_{k}=0,1, \ldots, N_{k}, N_{k} h_{k}=l_{k}, k=1,2, \ldots, d\right\}=\omega_{h}^{d} \cup \partial \omega_{h}^{d}, \\
\omega_{h}^{d}=\bar{\omega}_{h}^{d} \cap \Omega^{d}, \quad \partial \omega_{h}^{d}=\bar{\omega}_{h}^{d} \cap \partial \Omega^{d},
\end{gathered}
$$

we approximate problem (4.1)-(4.2) by the following difference scheme:

$$
\begin{gathered}
y_{t}=\Delta_{h} y^{2}+f, \quad(t, \mathbf{x}) \in \omega^{d+1}, \quad \Delta_{h} y=\sum_{k=1}^{d} y_{\bar{x}_{k} x_{k}}, \\
\left.\hat{y}\right|_{\partial \omega_{h}^{d}}=\hat{\mu}, \quad t \in \omega_{\tau} ; \quad y(0, \mathbf{x})=u_{0}(\mathbf{x}), \quad \mathbf{x} \in \bar{\omega}_{h}^{d} .
\end{gathered}
$$

Equation (4.4) can be rewritten in the form

$$
\hat{y}=\left(1-2 \tau \sum_{k=1}^{d} \frac{1}{h_{k}^{2}}\right) y+\sum_{k=1}^{d} \frac{\tau\left(y_{\left(-1_{k}\right)}^{2}+y_{\left(+1_{k}\right)}^{2}\right)}{h_{k}^{2}}+\tau f .
$$

Here

$$
y_{\bar{x}_{k} x_{k}}=\frac{y_{x_{k}}-y_{\bar{x}_{k}}}{h_{k}}, \quad y_{x_{k}}=\frac{y_{\left(+1_{k}\right)}-y}{h_{k}}, \quad y_{\bar{x}_{k}}=\frac{y-y_{\left(-1_{k}\right)}}{h_{k}} .
$$

Let us prove some properties of the grid operators.

Lemma 4.1. For any grid function given on the grid $\bar{\omega}_{h}$ the following inequality holds:

$$
\left(y^{2}\right)_{\bar{x}_{k} x_{k}} \geqslant 2 y y_{\bar{x}_{k} x_{k}} \text {. }
$$

Proof. Using the formula of the difference differentiation, we get

$$
\begin{aligned}
& \left(y^{2}\right)_{\bar{x}_{k} x_{k}}=\left(\left(y_{\left(+1_{k}\right)}+y\right) y_{\bar{x}_{k}}\right)_{x_{k}}=\left(y_{\left(+1_{k}\right)}+y\right) y_{\bar{x}_{k} x_{k}}+\left(y_{\bar{x}_{k}}+y_{x_{k}}\right) y_{\bar{x}_{k}}, \\
& \left(y^{2}\right)_{x_{k} \bar{x}_{k}}=\left(\left(y_{\left(+1_{k}\right)}+y\right) y_{x_{k}}\right)_{\bar{x}_{k}}=\left(y_{\left(-1_{k}\right)}+y\right) y_{\bar{x}_{k} x_{k}}+\left(y_{\bar{x}_{k}}+y_{x_{k}}\right) y_{x_{k}},
\end{aligned}
$$

Summing the last identities, we obtain

$$
\left(y^{2}\right)_{\bar{x}_{k} x_{k}}=\frac{y_{\left(+1_{k}\right)}+2 y+y_{\left(-1_{k}\right)}}{2} y_{\bar{x}_{k} x_{k}}+2 y_{\bar{x}_{k}}^{2} \geqslant 2 y y_{\bar{x}_{k} x_{k}}+0.5 h_{k}^{2} y_{\bar{x}_{k} x_{k}}^{2} \geqslant 2 y y_{\bar{x}_{k} x_{k}} .
$$

This completes the proof.

In particular, from (4.7) it follows that

$$
y_{t}=\Delta_{h} y^{2}+f \geqslant 2 y \Delta_{h} y .
$$

The discrete analogue of property $3^{\circ}$ ) is needed for the sequel. Using the Taylor series expansion of the grid function, we obtain

$$
\Delta_{h} u_{0}^{2}+f(0, \mathbf{x})=\Delta u_{0}^{2}+f(0, \mathbf{x})+\frac{1}{12} \sum_{k=1}^{d} h_{k}^{2} \frac{\partial^{4} \bar{u}_{k}^{2}}{\partial x_{k}^{4}} \leqslant-\varepsilon+\frac{h^{2} d \kappa_{3}}{12} \leqslant 0
$$

for sufficiently small $h \leqslant h_{0}^{(1)}=\sqrt{12 \varepsilon / d \kappa_{3}}$, where

$$
\kappa_{3}=\max _{1 \leqslant k \leqslant d}\left\|\frac{\partial^{4} u_{0}^{2}}{\partial x_{k}^{4}}\right\|_{C\left(\bar{\Omega}^{d}\right)}, \quad \bar{u}_{k}=u_{0}\left(x_{1}, x_{2}, \ldots, \bar{x}_{k}, \ldots, x_{d}\right), \quad \bar{x}_{k} \in\left(x_{k}-h_{k}, x_{k}+h_{k}\right) .
$$


Similarly, it can be proved that the discrete analogue of inequality (4.3)

$$
\Delta_{h} u_{0} \leqslant \varepsilon_{1}<0
$$

is satisfied for

$$
h \leqslant h_{0}^{(2)}=\sqrt{12 \varepsilon_{1} / d \kappa_{4}}, \quad \kappa_{4}=\max _{1 \leqslant k \leqslant d}\left\|\frac{\partial^{4} u_{0}}{\partial x_{k}^{4}}\right\|_{C\left(\bar{\Omega}^{d}\right)} .
$$

For the solution of problem (4.4)-(4.5) the following theorem holds.

Theorem 4.1. Suppose conditions $\left.1^{\circ}\right)-3^{\circ}$ ) are satisfied and

$$
\begin{gathered}
h \leqslant h^{(0)}=\sqrt{12 M / d \kappa}, \quad M=\max \left\{\varepsilon, \varepsilon_{1}\right\}, \quad \kappa=\max \left\{\kappa_{3}, \kappa_{4}\right\}, \\
0 \leqslant y \leqslant c_{2}, \quad \Delta_{h} y \leqslant 0, \quad 1-4 \tau c_{2} \sum_{k=1}^{d} \frac{1}{h_{k}^{2}} \geqslant 0, \\
c_{2}=\max \left\{\max _{1 \leqslant k \leqslant N}\left\|\mu^{k}\right\|_{C\left(\partial \omega_{h}^{d}\right)},\left\|u_{0}\right\|_{C\left(\omega_{h}^{d}\right)}\right\}+\sum_{k=1}^{N} \tau\left\|f^{k}\right\|_{C\left(\omega_{h}^{d}\right)} .
\end{gathered}
$$

Then

$$
0 \leqslant \hat{y} \leqslant c_{2}, \quad \Delta_{h} \hat{y} \leqslant 0 .
$$

Proof. The nonnegativity of the grid function $\hat{y}$ follows from the structure of the difference scheme (4.6), (4.5) and from the assumptions. To prove the boundedness of the solution $\hat{y} \leqslant c_{1}$, we use the maximum principle technique. The grid function $\hat{y}$ can reach its maximum either on the boundary

$$
\|\hat{y}\|_{C\left(\bar{\omega}_{h}^{d}\right)}=\|\hat{\mu}\|_{C\left(\partial \omega_{h}^{d}\right)}
$$

or at a certain interior point $\mathbf{x}^{*} \in \omega_{h}^{d}$

$$
\hat{y}^{*}=\max _{\mathbf{x} \in \omega_{h}} \hat{y}(t, \mathbf{x})=y\left(t, \mathbf{x}^{*}\right) .
$$

But then from the difference equation (4.6) it follows that

$$
\|\hat{y}\|_{C\left(\bar{\omega}_{h}^{d}\right)} \leqslant\left(1+\tau \Delta_{h} y^{*}\right)\|y\|_{C\left(\omega_{h}^{d}\right)}+\tau\|f\|_{C\left(\omega_{h}^{d}\right)} .
$$

Since $\Delta_{h} y \leqslant 0$, combining (4.15) and (4.16), we get

$$
\|\hat{y}\|_{C\left(\bar{\omega}_{h}^{d}\right)} \leqslant \max \left\{\|\hat{\mu}\|_{C\left(\partial \omega_{h}^{d}\right)},\|y\|_{C\left(\omega_{h}^{d}\right)}\right\}+\tau\|f\|_{C\left(\omega_{h}^{d}\right)} .
$$

The application of Lemma 2.3 yields $\hat{y} \leqslant c_{1}$. To prove the second condition of (4.14), we formulate the problem for the difference derivative $v=y_{t}$ in $t$. Using simple identity $\varphi=\tau \varphi_{\bar{t}}+\check{\varphi}$ and the representation of the difference scheme (4.4) in the form

$$
v=\Delta_{h} y^{2}+f,
$$

from (4.4), (4.5) we obtain

$$
\begin{gathered}
v=\check{v}+\tau \Delta_{h}((y+\hat{y}) v)+\tau f_{\bar{t}}, \\
v(0, \mathbf{x})=\Delta_{h} u_{0}^{2}+f(0, \mathbf{x}) \leqslant 0,\left.\quad v\right|_{\partial \omega_{h}^{d}}=\mu_{t} \leqslant 0 .
\end{gathered}
$$


Let us write the difference equation (4.18) in a more convenient form:

$$
v=\left(1-2 \tau(y+\check{y}) \sum_{k=1}^{d} \frac{1}{h_{k}}\right) \check{v}+\tau \sum_{k=1}^{d} \frac{y_{\left(-1_{k}\right)}+\check{y}_{\left(-1_{k}\right)}}{h_{k}^{2}} \check{v}_{\left(-1_{k}\right)}+\tau \sum_{k=1}^{d} \frac{y_{\left(+1_{k}\right)}+\check{y}_{\left(+1_{k}\right)}}{h_{k}^{2}} \check{v}_{\left(+1_{k}\right)}+\tau f_{\bar{t}} .
$$

Since the Courant - Friedrichs - Lewy condition (4.13) is satisfied, it follows that all coefficients of the difference equation (4.20) are positive; $v^{0} \leqslant 0$ by (4.14). Consequently, by induction it follows from (4.20) that $v^{k} \leqslant v^{n}$ for all $k \leqslant n$. Since we have proved that $0 \leqslant \hat{y} \leqslant c_{1}$, it follows from (4.20) that $v^{n+1} \leqslant 0$ for $k=n+1$. Hence, by (4.8) it follows that $\Delta_{h} \hat{y} \leqslant 0$.

The properties of the difference scheme (4.4), (4.5) are determined by the properties of the differential problem and by conditions $\left.1^{\circ}\right)-3^{\circ}$. So, we believe that to formulate the problem for the perturbation $\delta y=\tilde{y}-y$ and to study the stability of the difference scheme, it is more natural to perturb the input data of the differential problem and, subsequently, to approximate the perturbed differential problem. As a result, we construct the corresponding differential and difference problems

$$
\begin{gathered}
\frac{\partial \tilde{u}}{\partial t}=\Delta \tilde{u}^{2}+\tilde{f}, \quad 0<t \leqslant T, \quad \mathbf{x} \in \Omega^{d}, \\
\left.\tilde{u}(t, \mathbf{x})\right|_{\partial \Omega^{d}}=\tilde{\mu}(t, \mathbf{x}), \quad 0<t \leqslant T ; \quad \tilde{u}(0, \mathbf{x})=\tilde{u}_{0}(\mathbf{x}), \quad \mathbf{x} \in \bar{\Omega}^{d}, \\
\tilde{y}_{t}=\Delta_{h} \tilde{y}^{2}+\tilde{f}, \quad(t, \mathbf{x}) \in \omega^{d+1}, \\
\left.\hat{\tilde{y}}\right|_{\partial \omega_{h}^{d}}=\tilde{\tilde{\mu}}, \quad t \in \omega_{\tau} ; \quad \tilde{y}(0, \mathbf{x})=\tilde{u}_{0}(\mathbf{x}), \quad \mathbf{x} \in \bar{\omega}_{h}^{d} .
\end{gathered}
$$

Suppose that the solution $\tilde{u}(t, \mathbf{x})$ and the input data satisfy conditions similar to $\left.\left.1^{\circ}\right)-3^{\circ}\right)$. For the solution $\tilde{y}$ a theorem similar to Theorem 4.1 holds.

The problem for the perturbation $\delta y$ is of the form

$$
\begin{gathered}
\delta \hat{y}=\left(1-2 \tau(y+\tilde{y}) \sum_{k=1}^{d} \frac{1}{h_{k}^{2}}\right) \delta y+\tau \sum_{k=1}^{d} \frac{\left(y_{\left(-1_{k}\right)}+\tilde{y}_{\left(-1_{k}\right)}\right) \delta y_{\left(-1_{k}\right)}+\left(y_{\left(+1_{k}\right)}+\tilde{y}_{\left(+1_{k}\right)}\right) \delta y_{\left(+1_{k}\right)}}{h_{k}^{2}}+\tau \delta f, \\
\delta y^{0}=\delta u_{0}=\tilde{u}_{0}-u_{0},\left.\quad \delta y\right|_{\partial \omega_{h}^{d}}=\delta \mu=\tilde{\mu}-\mu .
\end{gathered}
$$

Let us note that under the conditions of Theorem 4.1 the coefficients of the right-hand side of (4.21) are positive and for $\delta \hat{y}$ the following estimate holds:

$$
\|\delta \hat{y}\|_{C\left(\bar{\omega}_{h}^{d}\right)} \leqslant \max \left\{\max _{1 \leqslant k \leqslant n}\left|\delta \mu^{k+1}\right|,\left\|\delta u_{0}\right\|_{C\left(\omega_{h}^{d}\right)}\right\}+\sum_{k=1}^{n} \tau\left\|\delta f^{k}\right\|_{C\left(\omega_{h}^{d}\right)} .
$$

The last inequality expresses the stability of the difference scheme with respect to a small perturbation of the problem input data. Note that estimate (4.23) is obtained for sufficiently small $h \leqslant h_{0}=\min \left\{h^{(0)}, \tilde{h}^{(0)}\right\}$ and under the Courant - Friedrichs - Lewy condition

$$
\begin{gathered}
1-2 \tau\left(c_{2}+\tilde{c}_{2}\right) \sum_{k=1}^{d} \frac{1}{h_{k}^{2}} \geqslant 0, \\
\tilde{c}_{2}=\max \left\{\max _{1 \leqslant k \leqslant N}\left\|\tilde{\mu}^{k}\right\|_{C\left(\partial \omega_{h}^{d}\right)},\left\|\tilde{u}_{0}\right\|_{C\left(\omega_{h}^{d}\right)}\right\}+\sum_{k=1}^{N} \tau\left\|\tilde{f}^{k}\right\|_{C\left(\omega_{h}^{d}\right)} .
\end{gathered}
$$

Under the conditions of Theorem 4.1 all coefficients of equation (4.21) are nonnegative. Hence, the considered difference scheme is monotone. 
Let us study the convergence of the solution of the difference scheme (4.4), (4.5) to the solution of the differential problem (4.1), (4.2). Consider the following difference scheme with respect to the exact solution $u=u(t, \mathbf{x})$ :

$$
\begin{gathered}
u_{t}=\Delta_{h} u^{2}+f+\psi, \quad(t, \mathbf{x}) \in \omega^{d}, \\
\left.\hat{u}\right|_{\partial \omega_{h}^{d}}=\hat{\mu}, \quad t \in \omega_{\tau}, \quad u(0, \mathbf{x})=u_{0}(\mathbf{x}), \quad \mathbf{x} \in \bar{\omega}_{h}^{d} .
\end{gathered}
$$

Here, for sufficiently smooth solution $u,\left\|\psi^{n}\right\|_{C\left(\omega_{h}^{d}\right)} \leqslant M\left(h^{2}+\tau\right)(M=$ const $>0)$ is the truncation error of the difference scheme. The problem for the error $e=y-u$ is of the form

$$
\begin{gathered}
\hat{e}=\left(1-2 \tau(y+u) \sum_{k=1}^{d} \frac{1}{h_{k}^{2}}\right) e+ \\
\tau \sum_{k=1}^{d} \frac{\left(y_{\left(-1_{k}\right)}+u_{\left(-1_{k}\right)}\right) e_{\left(-1_{k}\right)}+\left(y_{\left(+1_{k}\right)}+u_{\left(+1_{k}\right)}\right) e_{\left(+1_{k}\right)}}{h_{k}^{2}}+\tau \psi, \quad(t, \mathbf{x}) \in \omega^{d+1}, \\
\left.e\right|_{\partial \omega_{h}^{d}}=0, \quad t \in \omega_{\tau}, \quad e(0, \mathbf{x})=0, \quad \mathbf{x} \in \bar{\omega}_{h}^{d} .
\end{gathered}
$$

The application of the a priori estimate (4.23) to the solution of problem (4.27), (4.28) yields

$$
\|e\|_{C\left(\bar{\omega}_{h}^{d}\right)} \leqslant M t\left(h^{2}+\tau\right), \quad t \in \bar{\omega}_{\tau} .
$$

From (4.29) it follows that the difference scheme has the order of convergence $\mathcal{O}\left(h^{2}+\tau\right)$ provided that there exists a sufficiently smooth solution of the differential problem.

Remark 4.1. Also, similar results can be obtained for explicit difference schemes approximating initial-boundary value problems for the more general equation

$$
\begin{gathered}
\frac{\partial u}{\partial t}=\Delta F(u), \quad F^{\prime}(u) \geqslant \kappa_{0}>0 \\
F^{\prime \prime}(u) \geqslant 0, \quad \text { for } u \in \mathscr{D}_{u}=\left\{u(t, \mathbf{x}): 0<u_{1} \leqslant u(t, \mathbf{x}) \leqslant u_{2}, \quad(t, \mathbf{x}) \in \bar{Q}^{d+1}\right\} .
\end{gathered}
$$

In this case, the following identity is useful:

$$
\begin{gathered}
(\varphi(y))_{\bar{x} x}=\varphi_{\bar{y} \hat{y}}\left(y_{\dot{x}}\right)^{2}+\frac{1}{2}\left(\varphi_{y}+\varphi_{\bar{y}}\right) y_{\bar{x} x} \\
\varphi_{\bar{y} \hat{y}}=\frac{1}{0.5\left(y_{i+1}-y_{i-1}\right)}\left(\frac{\varphi\left(y_{i+1}\right)-\varphi\left(y_{i}\right)}{y_{i+1}-y_{i}}-\frac{\varphi\left(y_{i}\right)-\varphi\left(y_{i-1}\right)}{y_{i}-y_{i-1}}\right) .
\end{gathered}
$$

Acknowledgments. The authors would like to express their gratitude to professors A. A. Zlotnik and B. S. Jovanovich for useful discussions.

\section{References}

1. A. Amosov and A.Zlotnik, Difference scheme for equations of on-dimensional motion of viscous barotropic gas, Computational processes and systems, (1986), no. 4, pp. 192-218.

2. A. Amosov and A. Zlotnik, Difference schemes of second-order of accuracy for the equations of the one-dimensional motion of a viscous gas., U.S.S.R. Comput. Math. Math. Phys., 27 (1987), no. 4, pp. $46-57$.

3. P. Bergeret, Classification of smooth solutions to $2 \times 2$ hyperbolic systems with boundary damping., Math. Methods Appl. Sci., 20 (1997), no. 18, pp. 1563-1598. 
4. A. Bressan and H. Jenssen, On the convergence of Godunov scheme for nonlinear hyperbolic systems., Chin. Ann. Math., Ser. B, 21 (2000), no. 3, pp. 269-284.

5. R. Courant and D. Hilbert, Methods of mathematical physics. Vol. II: Partial differential equations., New York-London: Interscience Publishers, a division of John Wiley \& Sons. XXII, 1962.

6. V.Demidovich, On a stability criterion for difference equtions, Differ. Uravn., 5 (1969), no.7, pp. 1247-1255, in Russian.

7. V. A. Galaktionov and J. L. Vázquez, A stability technique for evolution partial differential equations. A dynamical systems approach., Progress in Nonlinear Differential Equations and their Applications 56. Boston, MA: Birkhäuser. xix, 2004.

8. E. Godlewski and P.-A. Raviart, Hyperbolic Systems of Conservation Law, Ellipses-Edition Marketing, 1991.

9. S. Godunov, Difference method for numerical calculation of discontinuous solutions of the fluid dynamics equations, Mat. Sb., N. Ser., 47(89) (1959), no. 3, pp. 273-306 (in Russian).

10. S. Klainerman and A. Majda, Formation of singularities for wave equations including the nonlinear vibrating string., Commun. Pure Appl. Math., 33 (1980), pp. 241-263.

11. S. V. Lemeshevsky, E. K. Makarov, and P. P. Matus, Bihari lemma and its application to investigation of stability of non-linear difference schemes, dokl. NAN Belarus, to appear, 2008.

12. R. J. Leveque, Finite volume methods for hyperbolic problems., Cambridge Texts in Applied Mathematics. Cambridge: Cambridge University Press. xix, 2002.

13. P. Lions, B. Perthame, and E. Tadmor, Kinetic formulation of the isentropic gas dynamics and psystems., Commun. Math. Phys., 163 (1994), no. 2, pp. 415-431.

14. A. Majda, Compressible fluid flow and systems of conservation laws in several space variables., Applied Mathematical Sciences, 53. New York etc.: Springer-Verlag. VIII, 1984.

15. P. Matus, The maximum principle and some of its applications., Comput. Methods Appl. Math., 2 (2002), no. 1, pp. 50-91.

16. P. Matus, Stability of difference schemes for nonlinear time-dependent problems, Comp. Meth. Appl. Math., 3 (2003), no. 2, pp. 313-329.

17. P. Matus and A. Kolodynska, Nonlinear stability of the difference schemes for equations of isentropic gas dynamics., Comput. Methods Appl. Math., 8 (2008), no. 2, pp. 155-170.

18. P. Matus and G. Marcinkiewicz, On the stability of a monotone difference scheme for the Burgers equation., Differ. Equ., 41 (2005), no. 7, pp. 1003-1009.

19. P. P. Matus, V. A. Irkhin, M. Lapinska-Chrzczonowicz, and S. V. Lemeshevsky, On exact difference schemes for hyperbolic and parabolic equations, Differ. Uravn., 43 (2007), no. 7, pp. 978-986 (in Russian).

20. V. Mazhukin, D. Malaphei, P. Matus, and A.Samarskij, Difference schemes on irregular grids for equations of mathematical physics with variable coefficients., Comput. Math. Math. Phys., 41 (2001), no.3, pp. 379-391.

21. V. Ostapenko, On the strong monotonicity of nonlinear difference schemes., Comput. Math. Math. Phys., 38 (1998), no. 7, pp. 1119-1133.

22. V. Ostapenko, Strong monotonicity of finite-difference schemes for systems of conservation laws., Comput. Math. Math. Phys., 39 (1999), no. 10, pp. 1619-1635.

23. M.Pinto, Integral inequalities of Bihari-type and applications, Funkcialaj Ekvacioj, 33 (1990), pp. $387-403$.

24. B. Rozhdestvenskij and N. Yanenko, Systems of quasilinear equations and their applications to gas dynamics. 2nd ed. (Sistemy kvazilinejnykh uravnenij $i$ ikh prilozehniya $k$ gazovoj dinamike)., Moskva: "Nauka". Glavnaya Redaktsiya Fiziko-Matematicheskoj Literatury, 1978.

25. A. Samarskii, The theory of difference schemes, Pure and Applied Mathematics, Marcel Dekker. 240. New York, NY: Marcel Dekker, 2001.

26. A. Samarskii and A. Gulin, Ustoicivost' raznostnyh shem, Nauka, Moscow, 1973 (in Russian).

27. A. Samarskii and P. Vabishchevich, Numerical methods for solution of convection-diffusion problems, URSS, Moscow, 1999.

28. A. Samarskii and P. Vabishchevich, Difference schemes for the transport equation., Dokl. Akad. Nauk, Ross. Akad. Nauk, 361 (2001), no. 1, pp. 21-23 (in Russian).

29. K. Vyaznikov, V. Tishkin, and A. Favorskij, Construction of monotone high resolution difference schemes for hyperbolic systems., Mat. Model., 1 (1989), no. 5, pp. 95-120. 